\title{
Water pair potential of near spectroscopic accuracy. II. Vibration-rotation-tunneling levels of the water dimer
}

\author{
G. C. Groenenboom, P. E. S. Wormer, and A. van der Avoird \\ Institute of Theoretical Chemistry, NSR-Center, University of Nijmegen, Toernooiveld, \\ 6525 ED Nijmegen, The Netherlands \\ E. M. Mas \\ Department of Physics and Astronomy, University of Delaware, Newark, Delaware 19716 \\ and Los Alamos National Laboratory, Los Alamos, New Mexico 87545 \\ R. Bukowski \\ Department of Physics and Astronomy, University of Delaware, Newark, Delaware 19716 \\ and Department of Chemistry, Quantum Chemistry Laboratory, University of Warsaw, Pasteura 1, \\ 02-093, Warsaw, Poland \\ K. Szalewicz \\ Department of Physics and Astronomy, University of Delaware, Newark, Delaware 19716
}

(Received 6 June 2000; accepted 28 July 2000)

\begin{abstract}
Nearly exact six-dimensional quantum calculations of the vibration-rotation-tunneling (VRT) levels of the water dimer for values of the rotational quantum numbers $J$ and $K \leqslant 2$ show that the SAPT-5s water pair potential presented in the preceding paper (paper I) gives a good representation of the experimental high-resolution far-infrared spectrum of the water dimer. After analyzing the sensitivity of the transition frequencies with respect to the linear parameters in the potential we could further improve this potential by using only one of the experimentally determined tunneling splittings of the ground state in $\left(\mathrm{H}_{2} \mathrm{O}\right)_{2}$. The accuracy of the resulting water pair potential, SAPT-5st, is established by comparison with the spectroscopic data of both $\left(\mathrm{H}_{2} \mathrm{O}\right)_{2}$ and $\left(\mathrm{D}_{2} \mathrm{O}\right)_{2}$ : ground and excited state tunneling splittings and rotational constants, as well as the frequencies of the intermolecular vibrations. (c) 2000 American Institute of Physics. [S0021-9606(00)30240-9]
\end{abstract}

\section{INTRODUCTION}

Very precise information about the pair potential of water can be extracted, in principle, from the microwave and (far-)infrared spectra of the water dimer ${ }^{1-17}$ prepared in supersonic molecular beams. Many-body interactions in water have also been probed by molecular-beam spectroscopy: high-resolution far-infrared spectra were recorded for the water trimer, tetramer, pentamer, and hexamer, ${ }^{18-25}$ mid- and near-infrared spectra for clusters up to the decamer. ${ }^{26-29}$

The temperature of these clusters is usually around $5 \mathrm{~K}$, which is so cold that only a few levels are thermally occupied. The observed lines in the high-resolution spectra correspond to transitions between the individual quantum levels of the clusters, with well-defined rotational quantum numbers $J$ and $K$ and with even minute tunneling splittings (less than $1 \mathrm{MHz}$ ) resolved. As the required Boltzmann averaging is only over a few occupied states, which are of specific permutation-inversion symmetry and have definite values of $J$ and $K$, the spectra can be completely assigned. The vibration-rotation-tunneling (VRT) levels extracted from the spectra indicate that the same kind of processes occur in clusters as in liquid water, ${ }^{30-32}$ namely, the breaking and reconstruction of hydrogen bonds. In these very cold clusters the bond breaking is solely due to quantum mechanical tunneling through the barriers in the potential separating multiple equivalent hydrogen bonded equilibrium structures. During the last few years it has become clear ${ }^{14,33-35}$ that the
VRT level splittings form an extremely sensitive probe of the detailed shape of the intermolecular potential surface. This is in contrast to the equilibrium structures of the small water clusters that can be predicted by rather simple model potentials. Hence, the dimer spectrum forms a very critical test of the pair potential in the region of the equivalent van der Waals wells and is also sensitive to the barriers separating those, while the trimer spectrum probes the three-body forces as well. Many potentials have been tested regarding their ability to predict VRT levels of the water dimer, cf. Figs. 4-6 in Ref. 14, Fig. 12 in Ref. 34, and trimer, cf. Fig. 6 in Ref. 36. However, all of these $a b$ initio and empirical water potentials produced VRT transition frequencies deviating from experiment by factors of 2,3 , or even by an order of magnitude. Hence, they are clearly not of spectroscopic accuracy. The only exception is the recent pair potential of Fellers et al. ${ }^{37}$ which was fit to the dimer spectrum.

The preceding paper ${ }^{38}$ presents a new pair potential for water obtained from $a b$ initio electronic structure calculations by symmetry-adapted perturbation theory (SAPT). In a recent communication ${ }^{35}$ it was briefly shown that the application of this SAPT-5s pair potential in rigorous quantum calculations of the VRT levels of the water dimer gives good agreement with the experimental high-resolution spectra. It was shown that the use of only one of the experimental ground state tunneling splittings for $\left(\mathrm{H}_{2} \mathrm{O}\right)_{2}$ to scale some of the parameters in the analytic representation of the SAPT- $5 \mathrm{~s}$ 
potential led to an improved version of this potential, named SAPT-5st, which gave excellent agreement with experiment for the frequencies of the intermolecular vibrations and tunneling splittings in both $\left(\mathrm{H}_{2} \mathrm{O}\right)_{2}$ and $\left(\mathrm{D}_{2} \mathrm{O}\right)_{2}$. Furthermore, it was demonstrated in Ref. 35 that with the addition of $a b$ initio calculated three-body interactions the SAPT-5s pair potential accurately reproduces the torsional levels of the water trimer. Here we report the method developed for the sixdimensional calculations of the VRT levels of the water dimer, as well as the scheme that was employed to utilize the experimental $\left(\mathrm{H}_{2} \mathrm{O}\right)_{2}$ spectrum for improvement of the $a b$ initio SAPT-5s potential. The VRT levels calculated for $\left(\mathrm{H}_{2} \mathrm{O}\right)_{2}$ and $\left(\mathrm{D}_{2} \mathrm{O}\right)_{2}$, which include the ground and excited state tunneling splittings, the intermolecular vibrational frequencies, and the rotational constants $A$ and $B+C$ of the dimer, are discussed and compared with experiment.

\section{TUNNELING AND VIBRATIONS IN THE WATER DIMER}

The equilibrium structure of the water dimer was predicted by $a b$ initio calculations ${ }^{39-42}$ and experimentally determined in 1974 by microwave spectroscopy. ${ }^{1}$ In 1977 it was shown by the now classical work of Dyke and co-workers ${ }^{2,43}$ that the six-dimensional intermolecular potential surface of the water dimer has eight equivalentpermutationally distinct—global minima and that this dimer may tunnel between the eight equivalent equilibrium structures. The equilibrium geometry has reflection symmetry and its point group $C_{s}$ is isomorphic to the permutation-inversion group $G_{2}=\left\{E,(12)^{*}\right\}$, with 1 and 2 labeling the two acceptor protons. Also the feasible permutation-inversion (PI) symmetry group $G_{16}$ associated with the actually observed tunneling processes was discussed ${ }^{43}$ already in 1977 . The VRT levels of the water dimer can be labeled by the irreducible representations (irreps) of this PI group.

Three different tunneling processes allow the dimer to interconvert between the eight minima, see Fig. 1. The first process, acceptor tunneling, does not require complete breaking of the hydrogen bond and has the lowest barrier: 156 $\mathrm{cm}^{-1}$ in the SAPT-5s potential. ${ }^{38}$ The permutation made feasible by acceptor tunneling is (12). This permutation corresponds to a simple rotation of the acceptor about its $C_{2}$ axis, but note that the minimum energy pathway for this tunneling process is actually more complicated. Acceptor tunneling yields a relatively large splitting between the $A_{1}^{ \pm}, E^{ \pm}, B_{1}^{ \pm}$ levels, on the one hand, and the $A_{2}^{\mp}, E^{\mp}, B_{2}^{\mp}$ levels, on the other. The magnitude of this splitting is about $10 \mathrm{~cm}^{-1}$ in $\left(\mathrm{H}_{2} \mathrm{O}\right)_{2}$ and about $2 \mathrm{~cm}^{-1}$ in $\left(\mathrm{D}_{2} \mathrm{O}\right)_{2}$, and it depends strongly on the value of the rotational quantum number $K$. The water dimer is a prolate near-symmetric rotor and $K$ is the projection of the total angular momentum $\mathbf{J}$ on the long axis- the $a$ axis. The second and third processes, donor-acceptor interchange and bifurcation tunneling, involve hydrogen bond breaking with higher barriers: 185 and $636 \mathrm{~cm}^{-1}$, respectively, in the SAPT-5s potential. The permutation associated with donor-acceptor interchange tunneling is $(A B)(1423)$ $=(A B)(13)(24)(12)$, i.e., the simultaneous interchange of the oxygen nuclei $A$ and $B$ and the protons 1,2 and 3,4 of the monomers $A$ and $B$, combined with the acceptor tunnel-
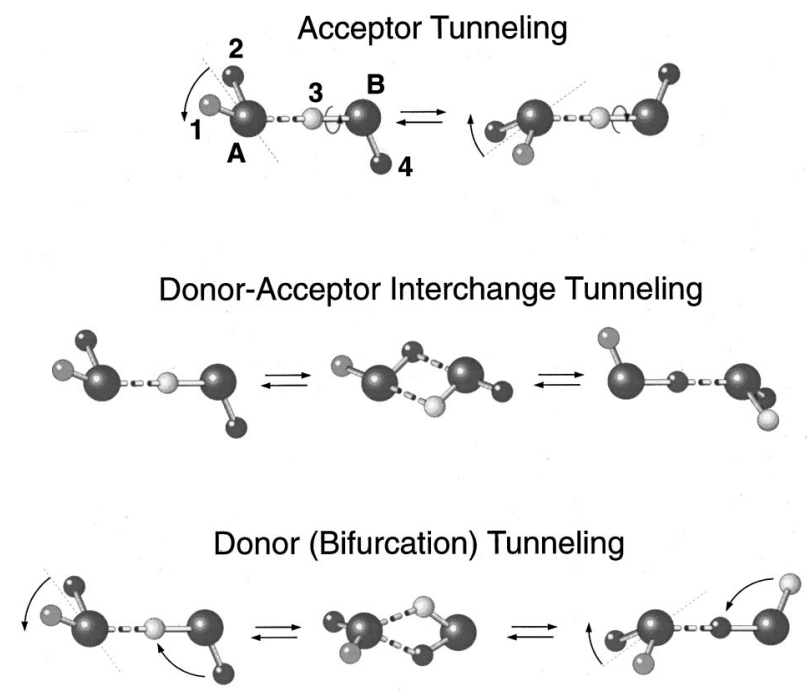

FIG. 1. Three different hydrogen bond rearrangement processes in the water dimer which connect the eight equivalent, permutationally distinct, equilibrium structures: acceptor tunneling with PI operation (12), donor-acceptor interchange tunneling with PI operation $(A B)(1423)$, and bifurcation (or donor) tunneling with PI operation (12)(34).

ing permutation (12). This process leads to splittings between the $A, E$, and $B$ levels which are typically $0.3 \mathrm{~cm}^{-1}$ in $\left(\mathrm{H}_{2} \mathrm{O}\right)_{2}$ and $0.02 \mathrm{~cm}^{-1}$ in $\left(\mathrm{D}_{2} \mathrm{O}\right)_{2}$. The permutation associated with bifurcation tunneling is (12)(34), where (34) exchanges the bound and free proton of the donor. This process does not cause a further splitting of the rovibrational levels, but leads to a shift of the $E$ levels relative to the $A$ and $B$ levels. ${ }^{44,45}$ This shift is very small: about $0.02 \mathrm{~cm}^{-1} \approx 700$ $\mathrm{MHz}$ for $\left(\mathrm{H}_{2} \mathrm{O}\right)_{2}$ and $7 \mathrm{MHz}$ for $\left(\mathrm{D}_{2} \mathrm{O}\right)_{2}$.

A detailed qualitative model that explains the tunneling splitting pattern of the water dimer levels in terms of a number of empirical parameters was developed by Coudert and Hougen. ${ }^{44,45}$ They used the water pair potential of Coker and Watts ${ }^{46}$ to find the three tunneling paths, and the internalaxis-method (IAM) ${ }^{47}$ to determine the amount of angular momentum generated by the tunneling motions and to derive the $J, K$ dependence of the splittings. This model was used as a basis to fit tunneling levels to the measured spectra; ${ }^{6-8}$ the result of this fit is a set of empirically determined parameters which completely determine all the ground state tunneling levels for arbitrary $J$ and $K$.

The excited intermolecular vibrations in the water dimer further probe the water pair potential. The six fundamental low-frequency vibrations of this type, see Fig. 7 in Ref. 14, are listed in Table I. The $A^{\prime}$ and $A^{\prime \prime}$ labels define the symmetry of these vibrations with respect to reflection in the point group $C_{s}$ of the equilibrium structure. Also the splitting of the vibrational levels into $G_{16}$ adapted tunneling components is indicated in Table I. The frequencies of these vibrations were calculated $a b$ initio in the harmonic approximation (for a recent calculation see Ref. 48), but it is clear from the nearly exact six-dimensional calculations in Refs. 14, 33, and 34 and in the present paper that the harmonic model is not valid in this case. It cannot represent the substantial splittings of the vibrational levels by tunneling between the eight equivalent minima, and the harmonic normal mode frequen- 
TABLE I. Intermolecular vibrations in the water dimer. This correspondence between $C_{s}$ irreps and $G_{16}$ irreps holds for pure vibrations, i.e., $J$ $=K=0$. The same relation extends to rovibrational states with $K=0$ and even values of $J$, while for $K=0$ and odd values of $J$ the labels $A^{\prime}$ and $A^{\prime \prime}$ must be reversed. For $K \neq 0$ one component of the $K$-doublet transforms as $A^{\prime}$ for even $J$ and as $A^{\prime \prime}$ for odd $J$, whereas the other component transforms as $A^{\prime \prime}$ for even $J$ and as $A^{\prime}$ for odd $J$ (Ref. 43).

\begin{tabular}{lccc}
\hline \hline \multicolumn{1}{c}{ Vibration } & Mode & $C_{s}$ irrep & $G_{16}$ irreps \\
\hline Stretch & $\nu_{7}$ & $A^{\prime}$ & $A_{1}^{+}, E^{+}, B_{1}^{+}, A_{2}^{-}, E^{-}, B_{2}^{-}$ \\
Acceptor wag & $\nu_{8}$ & $A^{\prime}$ & $A_{1}^{+}, E^{+}, B_{1}^{+}, A_{2}^{-}, E^{-}, B_{2}^{-}$ \\
In-plane bend & $\nu_{6}$ & $A^{\prime}$ & $A_{1}^{+}, E^{+}, B_{1}^{+}, A_{2}^{-}, E^{-}, B_{2}^{-}$ \\
Donor torsion & $\nu_{12}$ & $A^{\prime \prime}$ & $B_{1}^{-}, E^{-}, A_{1}^{-}, B_{2}^{+}, E^{+}, A_{2}^{+}$ \\
Acceptor twist & $\nu_{11}$ & $A^{\prime \prime}$ & $B_{1}^{-}, E^{-}, A_{1}^{-}, B_{2}^{+}, E^{+}, A_{2}^{+}$ \\
Out-of-plane bend & $\nu_{10}$ & $A^{\prime \prime}$ & $B_{1}^{-}, E^{-}, A_{1}^{-}, B_{2}^{+}, E^{+}, A_{2}^{+}$ \\
\hline \hline
\end{tabular}

cies (see Ref. 48 and the discussion below) are typically too high by a factor of 1.4 in comparison with the exact frequencies. ${ }^{14,16,17}$

\section{DYNAMICS CALCULATIONS}

\section{A. Theory}

Following a more approximate five-dimensional treatment by Althorpe and Clary ${ }^{49,50}$ in 1994 and some rigid-body quantum Monte Carlo calculations ${ }^{51}$ giving estimates of the tunneling splittings, Leforestier et al. ${ }^{33}$ were in 1997 the first to calculate nearly exactly the VRT levels of the water dimer from a six-dimensional potential. They implemented a split Wigner pseudospectral method. ${ }^{52}$ Somewhat later, the same problem was solved by Chen and Light ${ }^{34}$ with the use of a sequential diagonalization-truncation method which, up to now, is restricted to $J=0$. Both Leforestier et $a l .^{33,14}$ and Chen and Light ${ }^{34}$ used their method to test several ab initio and empirical water potentials against the dimer spectrum. Fellers et al. ${ }^{37}$ implemented the method of Leforestier et $a l^{33}$ as part of a fitting program, and obtained a spectroscopic water pair potential denoted by VRT(ASP-W). They started from the $a b$ initio based ASP-W potential of Millot and Stone ${ }^{53}$ and optimized some of the parameters in this potential through a fit of the dimer spectrum. ${ }^{37}$ Here, we present a very efficient implementation of the conventional variational method. We apply this method to test and improve the SAPT-5s water pair potential described in the preceding paper. ${ }^{38}$

All methods mentioned above start from the Hamiltonian of a rotating dimer consisting of two (rigid) polyatomic monomers, expressed in body-fixed dimer coordinates

$$
\begin{aligned}
H= & T_{A}+T_{B}+\frac{1}{2 \mu_{A B} R^{2}} \\
& \times\left[-\hbar^{2} \frac{\partial}{\partial R} R^{2} \frac{\partial}{\partial R}+J^{2}+j_{A B}^{2}-2 \mathbf{j}_{A B} \cdot \mathbf{J}\right] \\
& +V\left(R, \vartheta_{A}, \psi_{A}, \vartheta_{B}, \psi_{B}, \varphi_{A}-\varphi_{B}\right) .
\end{aligned}
$$

$R$ is the distance between the centers of mass of the monomers, while $\boldsymbol{\omega}_{A} \equiv\left(\varphi_{A}, \vartheta_{A}, \psi_{A}\right)$ and $\boldsymbol{\omega}_{B} \equiv\left(\varphi_{B}, \vartheta_{B}, \psi_{B}\right)$ are the Euler angles describing the orientations of the monomers $A$ and $B$ with respect to the dimer frame. The operator $\mathbf{J}$ represents the total angular momentum, $\mathbf{j}_{A B}=\mathbf{j}_{A}+\mathbf{j}_{B}$ is the sum of the monomer angular momenta, and $\mu_{A B}$ is the dimer reduced mass. The kinetic energy operator of monomer $X$ $(=A$ or $B)$ is given by

$$
T_{X}=A_{X}\left(j_{X x}^{\mathrm{BF}}\right)^{2}+B_{X}\left(j_{X y}^{\mathrm{BF}}\right)^{2}+C_{X}\left(j_{X z}^{\mathrm{BF}}\right)^{2},
$$

with the rotational constants $A_{X}, B_{X}$, and $C_{X}$. The superscript BF (body-fixed) implies that $x, y$, and $z$ refer to the components of $\mathbf{j}_{X}$ along the principal axes of monomer $X$. The Hamiltonian in Eq. (1) has been derived by Brocks et $a l .{ }^{54}$ with the use of the chain rule. An alternative derivation is given in Appendix A-4 of Ref. 55. It has also been applied in calculations of the VRT levels of the $\mathrm{NH}_{3}$ dimer. ${ }^{55-57}$

Just as in the earlier work on the $\mathrm{NH}_{3}$ dimer, ${ }^{55-57}$ we use a coupled product basis of symmetric rotor functionsWigner $D$-functions ${ }^{58}$ - for the angular coordinates

$$
\begin{aligned}
|\mathbf{I} ; J M\rangle= & {\left[\frac{\left(2 j_{A}+1\right)\left(2 j_{B}+1\right)(2 J+1)}{256 \pi^{5}}\right]^{1 / 2} } \\
& \times \sum_{m_{A} m_{B}} D_{m_{A} k_{A}}^{\left(j_{A}\right)}\left(\boldsymbol{\omega}_{A}\right)^{*} D_{m_{B} k_{B}}^{\left(j_{B}\right)}\left(\boldsymbol{\omega}_{B}\right)^{*} \\
& \times\left\langle j_{A} m_{A} ; j_{B} m_{B} \mid j_{A B} K\right\rangle D_{M K}^{(J)}(\alpha, \beta, 0)^{*}
\end{aligned}
$$

in which $\mathbf{I}=\left\{j_{A}, k_{A}, j_{B}, k_{B}, j_{A B}, K\right\}$ and $\left\langle j_{A} m_{A} ; j_{B} m_{B} \mid j_{A B} K\right\rangle$ is a Clebsch-Gordan coupling coefficient. ${ }^{58}$ The angles $(\alpha, \beta)$ are the polar angles of the intermolecular vector $\vec{R}$ $\equiv \vec{R}_{A B}$ with respect to the space-fixed frame. The same basis was used in Ref. 33, whereas Ref. 34 used a similar, but uncoupled, basis.

Both the potential and the kinetic energy are calculated in the basis of Eq. (3). The kinetic energy operator is diagonal in this basis, except for the monomer asymmetric rotor terms, cf. Eq. (2), and the small off-diagonal Coriolis coupling terms, see Ref. 55. For the radial basis $|n\rangle \equiv \Phi_{n}(R)$ we use a sinc function discrete variable representation (DVR). ${ }^{59,60}$ The sinc functions are contracted by coefficients obtained from the solution of a one-dimensional radial Schrödinger equation. As in the $\mathrm{NH}_{3}$ dimer work, ${ }^{55-57}$ the radial potential in this equation corresponds to a fixed-angles cut of the six-dimensional surface through the global minimum.

The potential is expanded in the same type of angular functions as the basis in Eq. (3). Since the potential is invariant under overall rotations, only the functions with $J=M$ $=K=0$ occur in its expansion, i.e., the expansion functions are 


$$
\begin{aligned}
A_{\Lambda}\left(\boldsymbol{\omega}_{A}, \boldsymbol{\omega}_{B}\right)= & \sum_{\substack{M_{A} \\
\left(M_{B}=-M_{A}\right)}}(-1)^{L_{A}+L_{B}+L} \\
& \times\left(\begin{array}{ccc}
L_{A} & L_{B} & L \\
M_{A} & M_{B} & 0
\end{array}\right) D_{M_{A} K_{A}}^{\left(L_{A}\right)}\left(\boldsymbol{\omega}_{A}\right)^{\star} \\
& \times D_{M_{B} K_{B}}^{\left(L_{B}\right)}\left(\boldsymbol{\omega}_{B}\right)^{\star}
\end{aligned}
$$

with $\Lambda \equiv\left\{L_{A}, L_{B}, L, K_{A}, K_{B}\right\}$. Note parenthetically that the phase $(-1)^{L_{A}+L_{B}+L}$ was inadvertently omitted in Ref. 55. The $R$-dependent expansion coeffients of the potential $V$ are

$$
\begin{aligned}
v_{\Lambda}(R)= & \frac{\left(2 L_{A}+1\right)\left(2 L_{B}+1\right)(2 L+1)}{64 \pi^{4}} \\
& \times \int d \boldsymbol{\omega}_{A} d \boldsymbol{\omega}_{B} A_{\Lambda}\left(\boldsymbol{\omega}_{A}, \boldsymbol{\omega}_{B}\right) V\left(R, \boldsymbol{\omega}_{A}, \boldsymbol{\omega}_{B}\right) .
\end{aligned}
$$

The potential energy matrix elements are given by

$$
\begin{aligned}
\left\langle n^{\prime}, \mathbf{I}^{\prime} ; J M|V| n, \mathbf{I} ; J M\right\rangle & \\
= & \delta_{K^{\prime} K} \sum_{\boldsymbol{\Lambda}} W\left(n^{\prime}, n, \boldsymbol{\Lambda}\right)(-1)^{j_{A}+j_{B}+j_{A B}+L_{A}+L_{B}-k_{A}^{\prime}-k_{B}^{\prime}-K} \\
& \times\left[\left(2 j_{A}^{\prime}+1\right)\left(2 j_{B}^{\prime}+1\right)\left(2 j_{A}+1\right)\left(2 j_{B}+1\right)\right. \\
& \left.\times\left(2 j_{A B}^{\prime}+1\right)\left(2 j_{A B}+1\right)\right]^{1 / 2} \\
& \times T_{A}\left(k_{A}^{\prime}, k_{A}, L_{A}\right) T_{B}\left(k_{B}^{\prime}, k_{B}, L_{B}\right) N\left(L_{A}, L_{B}, L\right),
\end{aligned}
$$

where the radial integrals are

$$
W\left(n^{\prime}, n, \boldsymbol{\Lambda}\right) \equiv\left\langle\Phi_{n^{\prime}}(R)\left|v_{\boldsymbol{\Lambda}}(R)\right| \Phi_{n}(R)\right\rangle
$$

and the angular integrals can be reduced to the following products of $3 j$ - and $9 j$-symbols ${ }^{55}$

$$
T_{X}\left(k_{X}^{\prime}, k_{X}, L_{X}\right) \equiv\left(\begin{array}{ccc}
j_{X}^{\prime} & L_{X} & j_{X} \\
-k_{X}^{\prime} & K_{X} & k_{X}
\end{array}\right), \quad X=A, B
$$

and

$$
N\left(L_{A}, L_{B}, L\right) \equiv\left(\begin{array}{ccc}
j_{A B}^{\prime} & L & j_{A B} \\
-K & 0 & K
\end{array}\right)\left\{\begin{array}{ccc}
j_{A}^{\prime} & L_{A} & j_{A} \\
j_{B}^{\prime} & L_{B} & j_{B} \\
j_{A B}^{\prime} & L & j_{A B}
\end{array}\right\} .
$$

The notations $T_{X}\left(k_{X}^{\prime}, k_{X}, L_{X}\right)$ and $N\left(L_{A}, L_{B}, L\right)$ are somewhat unusual. They refer to three-dimensional arrays in the algorithm described in Sec. III B and illustrated in Fig. 2. The basis was adapted to the $G_{16}$ symmetry, see Table II, and the calculations were performed for each $G_{16}$ irrep separately.

Before we describe our method in more detail and present the results, let us define the relevant quantities. In accordance with the recent experimental papers on the water $\operatorname{dimer}^{16,17}$ we use the expressions

$$
\begin{aligned}
& E_{1}=\left[E\left(A_{1}^{+}\right)+E\left(B_{1}^{+}\right)\right] / 2, \quad E_{2}=\left[E\left(A_{2}^{-}\right)+E\left(B_{2}^{-}\right)\right] / 2, \\
& i_{1}=E\left(B_{1}^{+}\right)-E\left(A_{1}^{+}\right), \quad i_{2}=E\left(B_{2}^{-}\right)-E\left(A_{2}^{-}\right) \\
& b_{1}=\left[E\left(E_{1}^{+}\right)-E_{1}\right] / 2, \quad b_{2}=\left[E\left(E_{2}^{-}\right)-E_{2}\right] / 2
\end{aligned}
$$

to define the donor-acceptor interchange splittings $i_{1}, i_{2}$ and the bifurcation tunneling shifts $b_{1}, b_{2}$ of the energy levels. The symbols $A_{1}^{+}, B_{1}^{+}, A_{2}^{-}, B_{2}^{-}$refer to the irreps of the group $G_{16}$, the levels $E\left(E_{1}^{+}\right)$and $E\left(E_{2}^{-}\right)$are levels of $E^{ \pm}$ symmetry that belong to a triplet with the levels of $A_{1}^{+}, B_{1}^{+}$ symmetry and with the levels of $A_{2}^{-}, B_{2}^{-}$symmetry, respectively. The $J, K$ dependence of the levels is given by the usual prolate near-symmetric top expression

$$
\begin{aligned}
E(J, K)= & E(J=0, K=0)+\frac{(B+C)}{2} J(J+1) \\
& +\left(A-\frac{B+C}{2}\right) K^{2}
\end{aligned}
$$

with the rotational constants $A, B, C$, but with neglect of the very small asymmetry doubling $(B-C) / 4$ (see below). These expressions define the origins $o_{1}=E_{1}(J=0, K=0)$ and $o_{2}=E_{2}(J=0, K=0)$ of the levels $E_{1}$ and $E_{2}$, respectively. Note that our $K$ corresponds to $K_{a}$ in Refs. 16 and 17. We also refer to the origins $o_{1}$ and $o_{2}$ of the levels with $K$

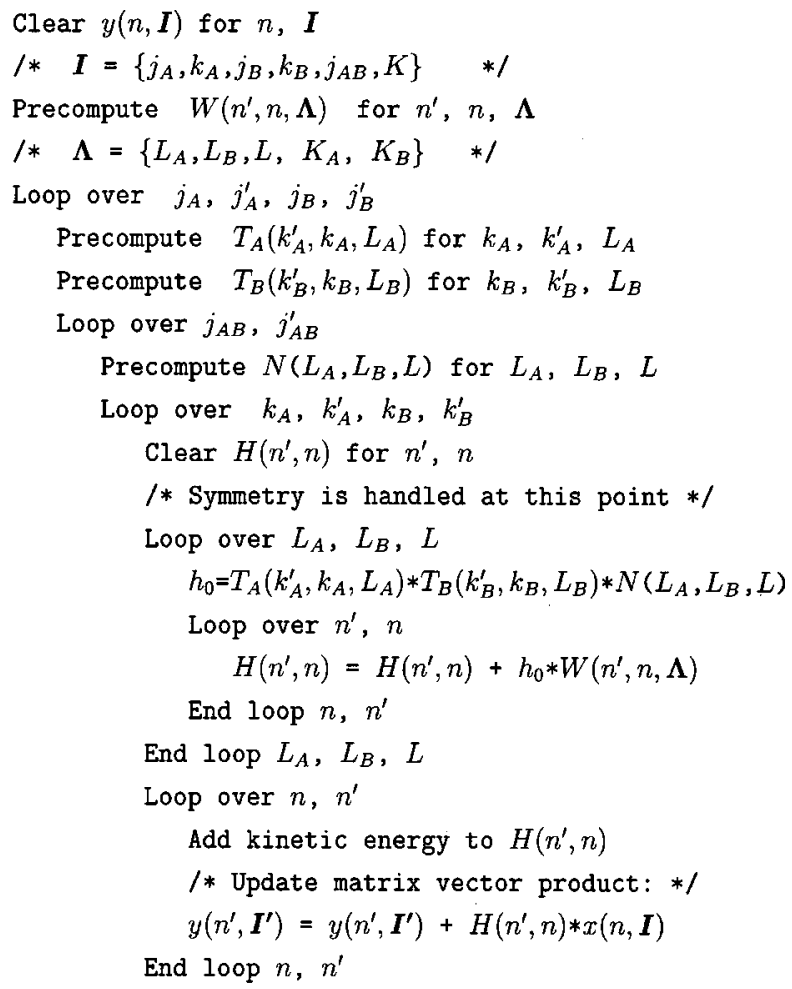

End loop $k_{A}, k_{A}^{\prime}, k_{B}, k_{B}^{\prime}$

End loop $j_{A B}, j_{A B}^{\prime}$

End loop $j_{A}, j_{A}^{\prime}, j_{B}, j_{B}^{\prime}$

FIG. 2. Schematic loop structure for the matrix vector multiply of Eq. (13). See Eqs. (7), (8), and (9) for the definition of $W\left(n^{\prime}, n, \boldsymbol{\Lambda}\right), T_{X}\left(k_{X}^{\prime}, k_{X}, L_{X}\right)$, and $N\left(L_{A}, L_{B}, L\right)$, respectively. The generation of symmetry-adapted matrix elements is not explicitly explained. Phases and other scalar factors are omitted. 
TABLE II. $G_{16}$ symmetry adapted linear combinations of basis functions. Here $\lambda \equiv J+j_{A}+j_{B}$. The last two columns give spin statistical weights. Note that functions with even $(e)$ or odd $(o) k$ values belong to different symmetry. The quantity $\bar{k}$ stands for $-k$.

\begin{tabular}{|c|c|c|c|c|c|}
\hline$\Gamma$ & $\begin{aligned} & \xi_{1}=\mid j_{A}, k_{A}, j_{B} \\
& \xi_{2}=\mid j_{B}, k_{B}, j_{A} \\
& \xi_{3}=\mid j_{A}, \bar{k}_{A}, j_{B} \\
& \xi_{4}=\mid j_{B}, \bar{k}_{B}, j_{A} \\
& k_{A}\end{aligned}$ & $\begin{array}{l}, K ; J, M\rangle \\
, \bar{K} ; J, M\rangle \\
\bar{K} ; J, M\rangle \\
, K ; J, M\rangle \\
k_{B}\end{array}$ & Linear combination & $\mathrm{w}_{\left(\mathrm{H}_{2} \mathrm{O}\right)_{2}}$ & $\mathrm{w}_{\left(\mathrm{D}_{2} \mathrm{O}\right)}$ \\
\hline$A_{1}^{+}$ & $e$ & $e$ & $\xi_{1}+(-1)^{\lambda} \xi_{2}+(-1)^{\lambda+j_{A B}} \xi_{3}+(-1)^{j_{A B}} \xi_{4}$ & 6 & 21 \\
\hline$A_{2}^{+}$ & $o$ & $o$ & $\xi_{1}-(-1)^{\lambda} \xi_{2}+(-1)^{\lambda+j_{A B}} \xi_{3}-(-1)^{j_{A B}} \xi_{4}$ & 0 & 3 \\
\hline$B_{1}^{+}$ & $e$ & $e$ & $\xi_{1}-(-1)^{\lambda} \xi_{2}+(-1)^{\lambda+j_{A B}} \xi_{3}-(-1)^{j_{A B}} \xi_{4}$ & 3 & 15 \\
\hline$B_{2}^{+}$ & $o$ & $o$ & $\xi_{1}+(-1)^{\lambda} \xi_{2}+(-1)^{\lambda+j_{A B}} \xi_{3}+(-1)^{j_{A B}} \xi_{4}$ & 1 & 6 \\
\hline$E_{x}^{+}$ & $e$ & $o$ & $\xi_{1}+(-1)^{\lambda+j_{A B}} \xi_{3}$ & 3 & 18 \\
\hline$E_{y}^{+}$ & $o$ & $e$ & $(-1)^{\lambda} \xi_{2}+(-1)^{j_{A B}} \xi_{4}$ & 3 & 18 \\
\hline$A_{1}^{-}$ & $e$ & $e$ & $\xi_{1}+(-1)^{\lambda} \xi_{2}-(-1)^{\lambda+j_{A B}} \xi_{3}-(-1)^{j_{A B}} \xi_{4}$ & 6 & 21 \\
\hline$A_{2}^{-}$ & $o$ & $o$ & $\xi_{1}-(-1)^{\lambda} \xi_{2}-(-1)^{\lambda+j_{A B}} \xi_{3}+(-1)^{j_{A B}} \xi_{4}$ & 0 & 3 \\
\hline$B_{1}^{-}$ & $e$ & $e$ & $\xi_{1}-(-1)^{\lambda} \xi_{2}-(-1)^{\lambda+j_{A B}} \xi_{3}+(-1)^{j_{A B}} \xi_{4}$ & 3 & 15 \\
\hline$B_{2}^{-}$ & $o$ & $o$ & $\xi_{1}+(-1)^{\lambda} \xi_{2}-(-1)^{\lambda+j_{A B}} \xi_{3}-(-1)^{j_{A B}} \xi_{4}$ & 1 & 6 \\
\hline$E_{x}^{-}$ & $e$ & $o$ & $\xi_{1}-(-1)^{\lambda+j_{A B}} \xi_{3}$ & 3 & 18 \\
\hline$E_{y}^{-}$ & $o$ & $e$ & $(-1)^{\lambda} \xi_{2}-(-1)^{j_{A B}} \xi_{4}$ & 3 & 18 \\
\hline
\end{tabular}

$=1$, which are the extrapolations of the levels $E_{1}(J, K=1)$ and $E_{2}(J, K=1)$ to $J=0$. The acceptor tunneling splitting $(a)$ is defined as

$$
a=o_{2}-o_{1} .
$$

\section{B. Computational method}

Because of the rather sharp minima in the water pair potential at the hydrogen bonded geometries and the occurrence of very small tunneling splittings and shifts, the calculations on the water dimer are much more demanding than those for the $\mathrm{NH}_{3}$ dimer. ${ }^{5-57}$ Truncation of the angular expansion of the potential in Eq. (4) beyond $L_{A}, L_{B}=7$ reduces the well depth $D_{e}=1759.0 \mathrm{~cm}^{-1}$ of the SAPT-5st potential (described below) to $D_{e}=1742.5 \mathrm{~cm}^{-1}$, while truncation beyond $L_{A}, L_{B}=8$ produces a well with $D_{e}=1763.1 \mathrm{~cm}^{-1}$, considerably better but slightly too deep. Fortunately, the effect of this truncation on the energy levels and the tunneling splittings, illustrated in Table III, is much smaller. It can be concluded from the results in this table that angular functions with $L_{A}$ and $L_{B}$ values up to 8 inclusive and 15789 terms in total are required to converge the expansion of the strongly anisotropic water pair potential.

The convergence of the energy levels with respect to truncation of the angular basis in Eq. (3) is also illustrated in Table III. The hardest to converge are the very small bifurcation shifts $b_{1}, b_{2}$ of the $E$ symmetry levels relative to the $A$ and $B$ levels of the same $A, E, B$ triplet, 1 or 2 . This is particularly difficult because the $E$ symmetry corresponds to mixed ortho/para dimers with $k_{A}, k_{B}=$ odd/even and the $A_{1}, B_{1}$ and $A_{2}, B_{2}$ levels to para/para and ortho/ortho dimers with $k_{A}, k_{B}=$ even/even and odd/odd, see Table II. These ortho/para designations hold for $\mathrm{H}_{2} \mathrm{O}$; for $\mathrm{D}_{2} \mathrm{O}$ they are interchanged. Hence, the convergence of the $A_{1}$ and $B_{1}$ levels shows a uniform behavior upon extension of the basis to higher $j_{A}, j_{B}$, and so does the convergence of the $A_{2}$ and $B_{2}$ levels, but the convergence of the $E_{1}$ and $E_{2}$ levels is nonuniform with respect to that of the $A_{1}, B_{1}$ and the $A_{2}, B_{2}$ levels. From the results in Table III we conclude that the inclusion of basis functions with $j_{A}, j_{B} \leqslant 11$ is just sufficient to converge the small bifurcation shifts for $\left(\mathrm{H}_{2} \mathrm{O}\right)_{2}$. For $\left(\mathrm{D}_{2} \mathrm{O}\right)_{2}$, where these bifurcation shifts are still smaller by two orders of magnitude, even the inclusion of functions with $j_{A}, j_{B}=12$ is not sufficient. All we can say here is that the computations are consistent with experiment because the calculated bifurcation shifts still decrease when the maximum $j_{A}, j_{B}$ is raised from 11 to 12 ; the results obtained with

TABLE III. Ground state $(J=K=0)$ VRT levels and tunneling splittings (in $\mathrm{cm}^{-1}$ ) from SAPT-5st.

\begin{tabular}{|c|c|c|c|c|}
\hline \multirow[b]{2}{*}{$\left(\mathrm{H}_{2} \mathrm{O}\right)_{2}$} & \multicolumn{2}{|c|}{$L_{A}, L_{B} \leqslant 7$} & \multicolumn{2}{|c|}{$L_{A}, L_{B} \leqslant 8$} \\
\hline & $j_{A}, j_{B} \leqslant 10$ & $j_{A}, j_{B} \leqslant 11$ & $j_{A}, j_{B} \leqslant 10$ & $j_{A}, j_{B} \leqslant 11$ \\
\hline$A_{1}^{+}$ & -1076.2898 & -1076.4308 & -1076.8643 & -1076.9946 \\
\hline$E^{+}$ & & & -1076.4312 & -1076.5979 \\
\hline$B_{1}^{+}$ & -1075.5516 & -1075.6957 & -1076.1419 & -1076.2784 \\
\hline$A_{2}^{-}$ & -1064.8820 & -1065.0390 & -1065.6333 & -1065.7819 \\
\hline$E^{-}$ & & & -1065.2540 & -1065.4246 \\
\hline$B_{2}^{-}$ & -1064.2147 & -1064.3739 & -1064.9825 & -1065.1360 \\
\hline$a$ & 11.37 & 11.36 & 11.19 & 11.18 \\
\hline$i_{1}$ & 0.738 & 0.735 & 0.722 & 0.716 \\
\hline$i_{2}$ & 0.667 & 0.665 & 0.651 & 0.646 \\
\hline$b_{1}$ & & & 0.036 & 0.019 \\
\hline \multirow[t]{2}{*}{$b_{2}$} & \multirow{2}{*}{\multicolumn{2}{|c|}{$L_{A}, L_{B} \leqslant 7$}} & 0.027 & 0.017 \\
\hline & & & \multicolumn{2}{|c|}{$L_{A}, L_{B} \leqslant 8$} \\
\hline$\left(\mathrm{D}_{2} \mathrm{O}\right)_{2}$ & \multicolumn{2}{|c|}{$j_{A}, j_{B} \leqslant 11$} & $j_{A}, j_{B} \leqslant 11$ & $j_{A}, j_{B} \leqslant 12$ \\
\hline$A_{1}^{+}$ & \multirow{2}{*}{\multicolumn{2}{|c|}{-1221.2152}} & -1222.6323 & -1222.9916 \\
\hline$E^{+}$ & & & -1222.6253 & -1222.9713 \\
\hline$B_{1}^{+}$ & \multicolumn{2}{|c|}{-1221.1718} & -1222.5940 & -1222.9512 \\
\hline$A_{2}^{-}$ & \multirow{2}{*}{\multicolumn{2}{|c|}{-1219.4365}} & -1220.9375 & -1221.2986 \\
\hline$E^{-}$ & & & -1220.9256 & -1221.2819 \\
\hline$B_{2}^{-}$ & \multicolumn{2}{|c|}{-1219.3958} & -1220.8990 & -1221.2606 \\
\hline$a^{2}$ & \multicolumn{2}{|c|}{1.777} & 1.695 & 1.692 \\
\hline$i_{1}$ & \multicolumn{2}{|r|}{0.0434} & 0.0383 & 0.0404 \\
\hline$i_{2}$ & \multirow{3}{*}{\multicolumn{2}{|c|}{0.0407}} & 0.0385 & 0.0380 \\
\hline$b_{1}$ & & & 0.0061 & 0.0005 \\
\hline$b_{2}$ & & & 0.0036 & 0.0011 \\
\hline
\end{tabular}


$j_{A}, j_{B} \leqslant 12$ confirm that these shifts are smaller than 0.001 $\mathrm{cm}^{-1}$. The experimental values for $\left(\mathrm{D}_{2} \mathrm{O}\right)_{2}$ are about $7 \mathrm{MHz}$ $\approx 0.00023 \mathrm{~cm}^{-1}$. We decided to truncate the basis at $j_{A}, j_{B}$ $\leqslant 11$, both for $\left(\mathrm{H}_{2} \mathrm{O}\right)_{2}$ and $\left(\mathrm{D}_{2} \mathrm{O}\right)_{2}$, which is sufficient to converge all relevant quantities except the minute bifurcation shifts in $\left(\mathrm{D}_{2} \mathrm{O}\right)_{2}$.

The radial integrals in Eq. (7) are computed on a DVR grid with $4 \leqslant R \leqslant 9$ bohr containing 49 equally spaced points. We found that the use of just three contracted DVR functions $\Phi_{n}(R)$ was sufficient to converge the energy levels.

The large values of $j_{A}$ and $j_{B}$ lead to basis sizes of more than 100000 . Therefore, we compute the eigenvalues and eigenvectors of the Hamiltonian matrix by a direct variant of the iterative Davidson algorithm. ${ }^{61}$ Direct means that we recompute the Hamiltonian matrix elements in each iteration to avoid storage of the full matrix. Since the expansion of the potential contains nearly 16000 terms, an efficient algorithm is essential. A simple-minded program structure where all $3 j$ and $9 j$ symbols are computed in the innermost loops would be very slow, but, on the other hand, precomputation of all the $3 j$ and particularly the $9 j$ symbols would lead to huge storage requirements. Even the retrieval of the precomputed symbols would be expensive. At the heart of the Davidson algorithm we find a multiplication of the $H$-matrix with a trial vector $\mathbf{x}$ :

$$
y\left(n^{\prime}, \mathbf{I}^{\prime}\right)=\sum_{n, \mathbf{I}} H\left(n^{\prime}, \mathbf{I}^{\prime} ; n, \mathbf{I}\right) x(n, \mathbf{I}) .
$$

In the loop structure for this matrix-vector multiplydetailed in Fig. 2-bra and ket quantum numbers are alternated and $3 j$ - and $9 j$-symbols are partially precomputed. We perform the computation of the $3 n j$-symbols inside our loops so that memory and CPU time requirements are optimally balanced. Detailed timing of the algorithm shows that only a few percent of the CPU time is spent on computing the $3 n j$ symbols. Let us reiterate that our program fully exploits the $G_{16}$ symmetry of the problem.

Since the calculations on the water dimer described in this paper refer only to low $J$ values $(\leqslant 2)$, we neglect the off-diagonal Coriolis coupling terms. These terms couple the wave functions with a given value of $K$ to those with $K \pm 1$, and they are manifest in the experimental spectra as the asymmetry doubling $(B-C) J(J+1) / 4$. From the experimental data ${ }^{6-8,11,13,16,17}$ it is evident that even the largest $(B$ $-C) / 4$ values, which occur for $K= \pm 1$, are only of the order of $20 \mathrm{MHz} \approx 0.0007 \mathrm{~cm}^{-1}$. Because the off-diagonal Coriolis coupling effects are so small and do not interfere with the small tunneling splittings, their omission is justified.

Finally we mention that we used for $\mathrm{H}_{2} \mathrm{O}$ the mass $18.010560 \mathrm{u}$ and the rotational constants $A=27.8806, B$ $=14.5216, C=9.2778 \mathrm{~cm}^{-1}$, while for $\mathrm{D}_{2} \mathrm{O}$ these numbers are $20.02291 \mathrm{u}, A=15.41997, B=7.27297$, and $C$ $=4.84528 \mathrm{~cm}^{-1} .{ }^{62}$

\section{TUNING OF THE POTENTIAL}

One will see in Sec. V that the ab initio SAPT-5s potential presented in paper $\mathrm{I}^{38}$ produces VRT levels of $\left(\mathrm{H}_{2} \mathrm{O}\right)_{2}$ which are generally in very good agreement with experi- ment, except for the (ground state) acceptor tunneling splitting $a(K=0)+a(K=1)$. Therefore, we developed a procedure to determine the smallest change in the ab initio SAPT-5s potential that corrects this discrepancy. As the linear parameters in the electrostatic, induction, and dispersion terms in SAPT-5s were determined by means of separate long-range calculations, we chose to change only the 40 linear parameters $a_{1}^{a b}, a_{2}^{a b}, a_{3}^{a b}$, and $a_{0}^{a b}=\exp \left(\alpha_{a b}\right)$ in the exponential functions that represent the $a-b$ site-site exchange repulsion and overlap effects, see Eqs. (1)-(3) as well as Tables VII and VIII in paper I. ${ }^{38}$ We consider the first order change $\Delta E_{k}$ in the energy levels with respect to a variation $\Delta \mathbf{p}$ in the potential parameters $\mathbf{p}=\left(a_{m}^{a b}\right)$. By restricting ourselves to the linear parameters we have, without approximation

$$
V(\mathbf{p}+\Delta \mathbf{p})=V(\mathbf{p})+\sum_{i} \frac{\partial V}{\partial p_{i}} \Delta p_{i}
$$

The wave functions $\psi_{k}$ corresponding to the energy levels $E_{k}$ are eigenfunctions of the Hamiltonian $H=T+V$ in a finite space spanned by a parameter-independent basis. Hence, we may apply the Hellmann-Feynman theorem, ${ }^{63}$ which gives in first order

$$
\Delta E_{k} \equiv E_{k}(\mathbf{p}+\Delta \mathbf{p})-E_{k}(\mathbf{p})=\sum_{i} A_{k i} \Delta p_{i},
$$

with

$$
A_{k i} \equiv\left\langle\psi_{k}\left|\frac{\partial V}{\partial p_{i}}\right| \psi_{k}\right\rangle .
$$

Since each of the splittings $s_{l}$ observed in the spectra is calculated as a difference between energy levels or between averages of energy levels, it can be written as a simple linear combination

$$
s_{l}=\sum_{k} C_{l k} E_{k}
$$

with constant coefficients $C_{l k}$. Thus, to first order

$$
\Delta s_{l} \equiv s_{l}(\mathbf{p}+\Delta \mathbf{p})-s_{l}(\mathbf{p})=\sum_{k} C_{l k} \sum_{i} A_{k i} \Delta p_{i},
$$

or, in matrix notation

$$
\Delta \mathbf{s}=\mathbf{C A} \Delta \mathbf{p} \equiv \mathbf{D} \Delta \mathbf{p} .
$$

If $s_{l}$ (observed) are the splittings actually extracted from the observed spectrum, we may put the following linear constraints on the parameter variation $\Delta \mathbf{p}$

$$
\mathbf{D} \Delta \mathbf{p}=\mathbf{s}(\text { observed })-\mathbf{s}(\mathbf{p}) \equiv \mathbf{d},
$$

where each row of the matrix $\mathbf{D}$ and each element of the column vector $\mathbf{d}$ correspond to one constraint. In the present work we impose only one linear constraint, namely that

$$
s_{1} \equiv a(K=0)+a(K=1)=\sum_{k} C_{1 k} E_{k}
$$

which is the sum of the $K=0$ and $K=1$ acceptor tunneling splittings for which we want to improve agreement with experiment becomes equal to the experimentally observed 
value of $13.92 \mathrm{~cm}^{-1}$. Hence, $d_{1}=13.92 \mathrm{~cm}^{-1}-s_{1}(\mathbf{p})$. The coefficients $C_{1 k}$ follow immediately from the definition of $a(K)$ in Eqs. (10)-(12).

The condition of minimal change in the potential implies that we minimize

$$
B(\Delta \mathbf{p})=\int_{4}^{9} R^{2} d R \int d \boldsymbol{\omega}_{A} d \boldsymbol{\omega}_{B}[V(\mathbf{p}+\Delta \mathbf{p})-V(\mathbf{p})]^{2},
$$

where the radial integrals are evaluated on the DVR grid with $4 \leqslant R \leqslant 9$ bohr described in Sec. III B. For $R>9$ bohr the change in the potential will be minimal anyway, since we exclude the long-range parameters from the tuning process. For $R<4$ bohr the potential is strongly repulsive and changes in this region are irrelevant for the present work. Using Eq. (14) we may rewrite Eq. (22) as

$$
B(\Delta \mathbf{p})=\Delta \mathbf{p}^{T} \mathbf{S} \Delta \mathbf{p},
$$

where

$$
S_{i j}=N \int_{4}^{9} R^{2} d R \int d \boldsymbol{\omega}_{A} d \boldsymbol{\omega}_{B} \frac{\partial V}{\partial p_{i}} \frac{\partial V}{\partial p_{j}}
$$

is a positive definite matrix. The normalization factor $N$ is chosen such that $\|\mathbf{S}\|=1$, where the norm of a matrix is defined as its largest singular value. All quantities are expressed in atomic units.

The problem of minimizing $B$ [Eq. (23)] with the linear constraints of Eq. (20) can be solved by the Lagrange multiplier method. This leads to the formal solution

$$
\Delta \mathbf{p}=\mathbf{S}^{-1} \mathbf{D}^{T}\left(\mathbf{D S}^{-1} \mathbf{D}^{T}\right)^{-1} \mathbf{d} .
$$

However, the matrix $\mathbf{S}$ is nearly singular. In the problem at hand its condition number is on the order of $10^{19}$. Appendix A shows how to solve these equations by the generalized singular value decomposition method, which is a numerically much more stable procedure than the standard matrix inversion. An efficient algorithm to compute the matrix $\mathbf{A}$ defined in Eq. (16) is also described in this Appendix.

Substitution of $\Delta \mathbf{p}$ thus obtained into Eq. (15) gives the first-order estimate $\Delta E_{k}$ of the change in all energy levels. We found that the change predicted for most quantities with just the constraint that $s_{1}=13.92 \mathrm{~cm}^{-1}$ and the minimization of $B$ in Eq. (22) was quite acceptable, except for the lower and upper donor-acceptor interchange splittings $i_{1}, i_{2}$ which became too large. We could have added the changes in these quantities as strict first order constraints in Eq. (20). However, each constraint reduces the number of degrees of freedom left to minimize the change in the potential. Therefore, we decided to minimize the changes of the splittings $i_{1}$ and $i_{2}$ from their $a b$ initio values rather than force them to agree with experiment. We imposed these conditions as soft constraints by adding the quadratic cost term

$$
\left|\Delta s_{l}\right|^{2}=\Delta \mathbf{p}^{T} \mathbf{S}^{(l)} \Delta \mathbf{p}
$$

to Eq. (23), which must be minimized. Here

$$
S_{i j}^{(l)}=N^{\prime} \sum_{k k^{\prime}} C_{l k} A_{k i} C_{l k^{\prime}} A_{k^{\prime} j}
$$

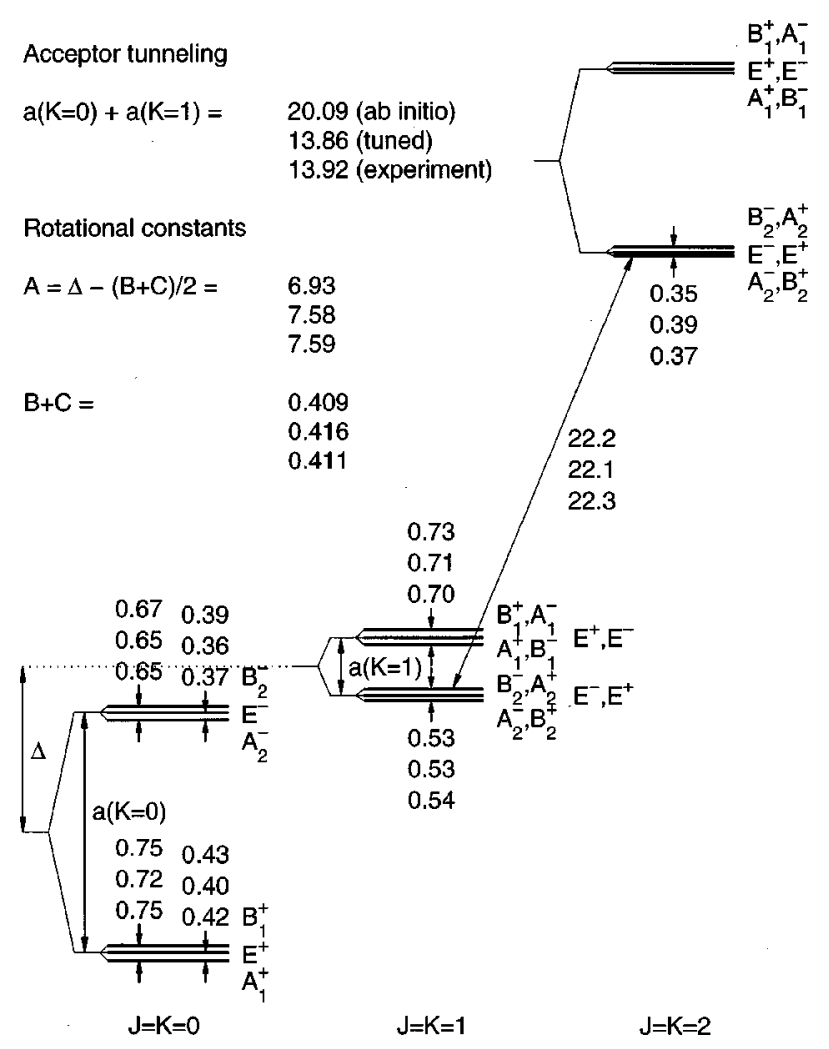

FIG. 3. Ground state VRT levels of the $\mathrm{H}_{2} \mathrm{O}$ dimer (in $\mathrm{cm}^{-1}$ ) from converged calculations with the SAPT-5s $a b$ initio potential (upper numbers) and the tuned version of this potential (middle numbers), in comparison with experimental data (Refs. 7, 8, and 17) (lower numbers). The energies are drawn to scale.

The factor $N^{\prime}$ is chosen such that $\left\|\mathbf{S}^{(2)}+\mathbf{S}^{(3)}\right\|=1$. The coefficients $C_{l k}$ again follow immediately from the definitions of the lower $(l=2)$ and upper $(l=3)$ donor-acceptor interchange splittings $i_{1}$ and $i_{2}$ [Eq. (10)].

\section{DIMER VRT LEVELS}

Figure 3 shows the vibrational ground state VRT levels and tunneling splittings of the $\mathrm{H}_{2} \mathrm{O}$ dimer calculated from the $a b$ initio SAPT-5s potential for $J, K \leqslant 2$ and the experimental data. ${ }^{7,8,17}$ The smaller splittings resulting from the donoracceptor interchange and bifurcation tunneling are in remarkably good-within $0.03 \mathrm{~cm}^{-1}$-agreement with experiment, for each $J, K$. Also the end-over-end rotational constant $B$ $+C$, which is a measure for the average intermolecular distance $R$, and even the rotational constant $A$, which depends sensitively on the average orientations of the molecules in the dimer, are close to the measured values. The frequency of the $22.3 \mathrm{~cm}^{-1}$ transition observed between the lowest $K$ $=1$ and $K=2$ levels agrees with experiment to $0.1 \mathrm{~cm}^{-1}$. The bifurcation shifts which determine the positions of the $E$ levels relative to the $A$ and $B$ levels within the same triplet can be obtained by subtraction, see Eq. (10). Even these very small quantities (listed in Table IV) agree well with the experimental values: $b_{1}=0.023$ and $b_{2}=0.025 \mathrm{~cm}^{-1}$ (for $K$ $=0)$. The acceptor tunneling splittings $a(K)$ have not been directly measured, but the sum $a(K=0)+a(K=1)$ is known. This is the only quantity that is not so well repro- 
TABLE IV. Effect of tuning the SAPT-5s potential on tunneling splittings and rotational constants of $\left(\mathrm{H}_{2} \mathrm{O}\right)_{2}\left(\right.$ in cm $\left.^{-1}\right)$. The label $1^{\text {st }}$ order refers to the first order estimate [Eqs. (15) and (16)] of the effects of the parameter change $\Delta \mathbf{p}$ that yields the SAPT-5st potential, computed with the wave functions $\psi_{k}$ obtained from the SAPT-5s potential.

\begin{tabular}{lccc}
\hline \hline & SAPT-5s & $1^{\text {st }}$ order & SAPT-5st \\
\hline$D_{0}\left(A_{1}^{+}\right)$ & 1067.1 & 1071.9 & 1077.0 \\
$a(K=0)$ & 16.19 & 11.28 & 11.18 \\
$a(K=1)$ & 3.90 & 2.65 & 2.68 \\
$a(K=0)+a(K=1)$ & 20.09 & 13.92 & 13.86 \\
$B+C$ & 0.4089 & 0.4089 & 0.4166 \\
$A$ & 6.93 & 7.67 & 7.58 \\
& & & \\
$K=0$ & & & \\
$i_{1}$ & 0.75 & 0.74 & 0.72 \\
$i_{2}$ & 0.67 & 0.69 & 0.65 \\
$b_{1}$ & 0.025 & 0.024 & 0.019 \\
$b_{2}$ & 0.027 & 0.014 & 0.017 \\
& & & \\
$K=1$ & & & \\
$i_{1}$ & 0.73 & 0.78 & 0.71 \\
$i_{2}$ & 0.53 & 0.53 & 0.53 \\
$b_{1}$ & -0.023 & -0.017 & -0.017 \\
$b_{2}$ & -0.029 & -0.019 & -0.022 \\
\hline \hline
\end{tabular}

duced by the $a b$ initio SAPT-5s potential: it is overestimated by about $40 \%$. Model calculations ${ }^{64}$ indicate that this might be partly due to the assumption that the water molecules are rigid. From a comparison with the VRT levels obtained ${ }^{14,34}$ with water pair potentials previously derived from $a b$ initio calculations, it may be concluded that the SAPT-5s potential represents a significant improvement.

The corresponding levels of the $\mathrm{D}_{2} \mathrm{O}$ dimer are displayed in Fig. 4. The acceptor tunneling splittings are about six times smaller than in the $\mathrm{H}_{2} \mathrm{O}$ dimer, and the interchange splittings are smaller by factors of 10 to 20 . Nevertheless, the SAPT-5s potential produces interchange splittings that overestimate the experimental values only by about $20 \%$. Also the rotational constants $B+C$ and $A$ agree well with experiment, ${ }^{6,11,13,16}$ but the acceptor tunneling splittings deviate more strongly, just as in the $\mathrm{H}_{2} \mathrm{O}$ dimer. Here they are overestimated by a factor of 2 . However, one must keep in mind that the smaller the splittings, the more sensitive they are to the shape of the barriers in the potential.

We also performed calculations of the VRT levels of $\left(\mathrm{H}_{2} \mathrm{O}\right)_{2}$ with the SAPT-pp potential, ${ }^{65}$ which might be considered as the predecessor of the SAPT-5s potential, see paper $\mathrm{I}^{38}$ The acceptor tunneling splitting is overestimated by about the same amount as with SAPT-5s, but the smaller interchange and bifurcation tunneling splittings, although substantially better than with any previous water pair potential derived from $a b$ initio calculations,,${ }^{14,34}$ are definitely not of the same high quality as with the SAPT-5s potential.

Section IV describes the procedure that we developed to improve the SAPT-5s potential by using the experimental ground state VRT levels of $\left(\mathrm{H}_{2} \mathrm{O}\right)_{2}$. The only experimental quantity actually used in this tuning procedure is the (by SAPT-5s overestimated) acceptor tunneling splitting $a(K$ $=0)+a(K=1)$. The interchange splittings $i_{1}$ and $i_{2}$ were accurately represented by the ab initio SAPT-5s potential.

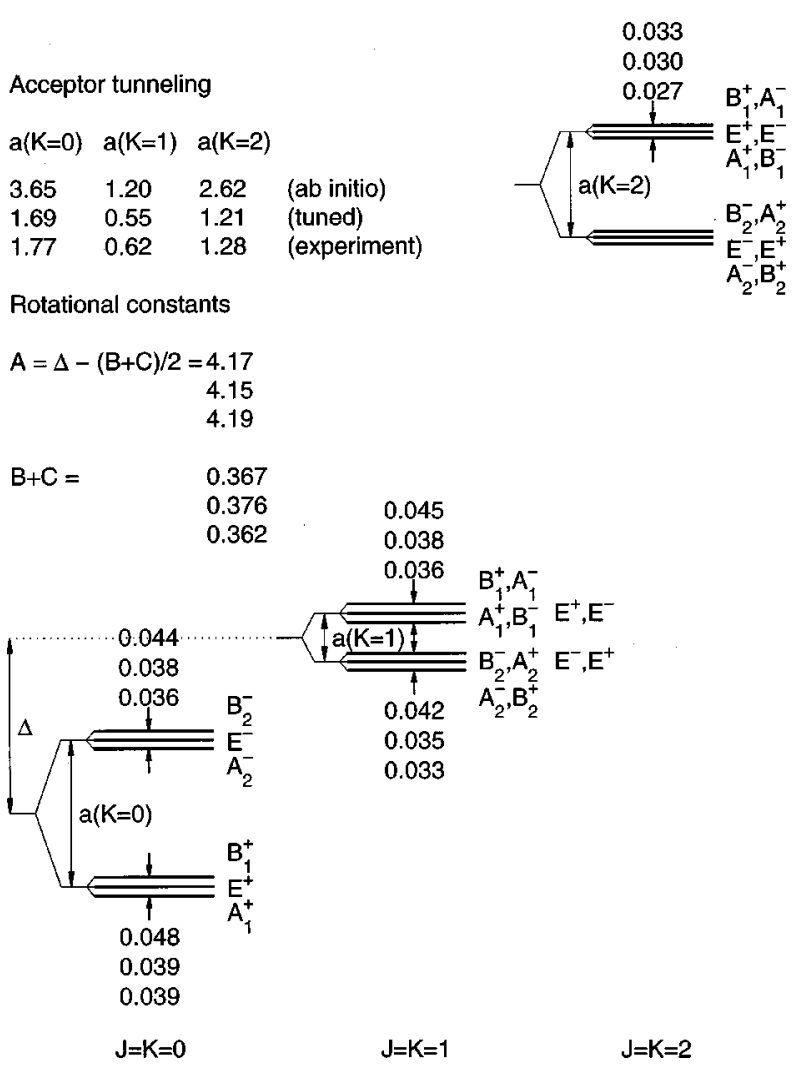

FIG. 4. Ground state VRT levels of the $\mathrm{D}_{2} \mathrm{O}$ dimer (in $\mathrm{cm}^{-1}$ ) calculated from the SAPT-5s ab initio potential (upper numbers) and from the tuned version of this potential (middle numbers), in comparison with experimental data (Refs. 6, 11, 13, and 16) (lower numbers). The energies are drawn to scale, except for the small interchange splittings which are enlarged by a factor of 10 .

The second soft constraint imposed in the tuning procedure in Sec. IV was that they change as little as possible. The first soft constraint applied was that the parameter modification leaves the potential as close as possible to the ab initio potential. Possible small effects of the nonrigidity of the water molecules are implicitly included by this tuning of the potential. Let us stress that the tuning was just a one-step procedure, i.e., the parameters computed from Eq. (25), $\mathbf{p}+\Delta \mathbf{p}$, determine the tuned potential. The quantity $\Delta \mathbf{p}$ can be directly used to get first-order estimates of all the spectroscopic quantities via Eq. (18). If, however, the VRT levels are recalculated with the tuned potential, the results will be different from the first-order approximation. In Table IV we show the first-order estimate of the change for several quantitites, as well as the actual change found after full recalculation of all the energy levels with the reparametrized SAPT-5s potential (referred to as SAPT-5st). Note that the estimate is very good, even for the acceptor tunneling where the largest change occurs. For the rotational constant $B+C$ the first order change is almost zero, because the internal part of the wave function is very nearly $J$ independent (for fixed $K$ ).

The VRT levels of $\left(\mathrm{H}_{2} \mathrm{O}\right)_{2}$ computed with SAPT-5st are in excellent agreement with experiment, see Fig. 3. Not only has the acceptor splitting $a(K=0)+a(K=1)$ been improved, but the rotational constant $A$ is now even better than with SAPT-5s, while the donor-acceptor interchange splittings $i_{1}$ and $i_{2}$ remain very close to the experimental values. 
TABLE V. VRT levels, tunneling splittings, and rotational constants $\left(\right.$ in $\left.\mathrm{cm}^{-1}\right)$ of $\left(\mathrm{H}_{2} \mathrm{O}\right)_{2}$ calculated from SAPT-5st. The numbers in parentheses are experimental values from Refs. 7, 8, 17, and 71. The assignment of the modes follows Refs. 17 and 71.

\begin{tabular}{|c|c|c|c|c|c|c|c|}
\hline & $o_{1}$ & $o_{2}{ }^{\mathrm{a}}$ & $a$ & $i_{1}$ & $i_{2}$ & $B+C$ & $A$ \\
\hline \multicolumn{8}{|c|}{ Ground state $\left(A^{\prime}\right)$} \\
\hline$K=0$ & $\begin{array}{c}0 \\
(0)\end{array}$ & $\begin{array}{c}11.18 \\
(11.18)\end{array}$ & $\begin{array}{r}11.18 \\
\left({ }^{b}\right)\end{array}$ & $\begin{array}{c}0.72 \\
(0.75)\end{array}$ & $\begin{array}{c}0.65 \\
(0.65)\end{array}$ & $\begin{array}{c}0.4166 \\
(0.4082)\end{array}$ & \\
\hline$K=1$ & $\begin{array}{c}14.30 \\
(14.39)\end{array}$ & $\begin{array}{c}11.62 \\
(11.66)\end{array}$ & $\begin{array}{r}2.68 \\
\left({ }^{b}\right)\end{array}$ & $\begin{array}{c}0.71 \\
(0.70)\end{array}$ & $\begin{array}{c}0.53 \\
(0.54)\end{array}$ & $\begin{array}{c}0.4165 \\
(0.4124)\end{array}$ & $\begin{array}{c}7.58 \\
(7.59)\end{array}$ \\
\hline \multicolumn{8}{|c|}{ Donor torsion $\left(A^{\prime \prime}\right)$} \\
\hline$K=0$ & 118.26 & $\begin{array}{c}64.35 \\
(64.52)^{\mathrm{c}}\end{array}$ & 53.91 & 3.05 & $\begin{array}{l}1.72 \\
(2.54)^{\mathrm{c}}\end{array}$ & 0.4174 & \\
\hline$K=1$ & $\begin{array}{c}87.13 \\
(87.75)\end{array}$ & 94.88 & 7.75 & $\begin{array}{c}0.45 \\
(1.11)\end{array}$ & 2.50 & $(0.4083)$ & -0.39 \\
\hline \multicolumn{8}{|c|}{ Acceptor wag $\left(A^{\prime}\right)$} \\
\hline$K=0$ & $\begin{array}{c}113.39 \\
(107.93)\end{array}$ & $\begin{array}{c}121.75 \\
(108.89)\end{array}$ & $\begin{array}{c}8.36 \\
(0.96)\end{array}$ & $\begin{array}{l}2.60 \\
(2.95)\end{array}$ & $\begin{array}{c}0.65 \\
(0.02)\end{array}$ & $\begin{array}{c}0.4138 \\
(0.4094)\end{array}$ & \\
\hline$K=1$ & $\begin{array}{c}118.54 \\
(109.98)\end{array}$ & $\begin{array}{c}131.08 \\
(123.56)\end{array}$ & $\begin{array}{c}12.53 \\
(13.58)\end{array}$ & $\begin{array}{c}7.27 \\
(5.24)\end{array}$ & $\begin{array}{c}4.77 \\
(3.41)\end{array}$ & $(0.4122)$ & $\begin{array}{c}7.45 \\
(8.01)\end{array}$ \\
\hline \multicolumn{8}{|c|}{ Acceptor twist $\left(A^{\prime \prime}\right)$} \\
\hline$K=0$ & 132.43 & $\begin{array}{c}120.97 \\
(120.19)\end{array}$ & 11.46 & 4.99 & $\begin{array}{l}10.36 \\
(9.39)\end{array}$ & $\begin{array}{c}0.4165 \\
(0.4138)\end{array}$ & \\
\hline$K=1$ & 147.08 & 137.80 & 9.28 & 4.34 & 6.96 & & 15.95 \\
\hline \multicolumn{8}{|c|}{ In-plane bend $\left(A^{\prime}\right)$} \\
\hline$K=0$ & 134.37 & $\begin{array}{l}156.46 \\
(114.37 ?)^{\mathrm{d}}\end{array}$ & 22.08 & 8.25 & 0.56 & $\begin{array}{l}0.4120 \\
(0.4171 ?)^{\mathrm{d}}\end{array}$ & \\
\hline$K=1$ & 160.78 & $\begin{array}{l}159.40 \\
(134.66 ?)^{\mathrm{d}}\end{array}$ & 1.38 & 0.85 & 0.29 & $(0.4104 ?)^{\mathrm{d}}$ & 14.88 \\
\hline
\end{tabular}

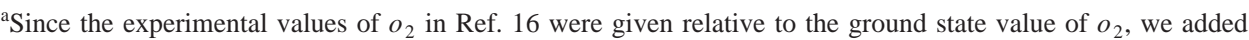
the estimated ground state acceptor splitting $a(K=0)=11.18 \mathrm{~cm}^{-1}$ to all experimental values.

${ }^{\mathrm{b}}$ Acceptor splitting $a(K=0)+a(K=1)=13.86$ (calculated) and 13.92 (experimental).

${ }^{\mathrm{c}}$ This band was not yet measured in Ref. 17; the experimental value (Ref. 71) is more recent than our prediction.

${ }^{\mathrm{d}}$ The question marks indicate doubts on the correctness of the assignment of the measured lines to the in-plane bending mode, see text.

The end-over-end rotational constant $B+C$ becomes slightly too large, indicating that the $R_{e}$ value of SAPT-5st is somewhat too small. The $K=0$ bifurcation shifts have become $b_{1}=0.019$ and $b_{2}=0.017 \mathrm{~cm}^{-1}$, still close to the experimental values $b_{1}=0.023$ and $b_{2}=0.025 \mathrm{~cm}^{-1}$, but it should be remembered that these small shifts are only marginally converged. The tuned potential was then used to compute the energy levels of $\left(\mathrm{D}_{2} \mathrm{O}\right)_{2}$ without any further reparametrization. As can be seen in Fig. 4, the results for $\left(\mathrm{D}_{2} \mathrm{O}\right)_{2}$ are markedly improved by the tuning. The $100 \%$ deviation from experiment for the acceptor tunneling splitting is reduced to $6 \%$ and the smaller $(20 \%)$ deviations of the interchange tunneling splittings are diminished to about 5\%. The VRT levels of $\left(\mathrm{D}_{2} \mathrm{O}\right)_{2}$ calculated from the SAPT-5st potential agree equally well with the experimental data as the results obtained from the VRT(ASP-W) potential, ${ }^{37}$ which was fit to these levels, while the representation of the $\left(\mathrm{H}_{2} \mathrm{O}\right)_{2}$ levels is better with the SAPT-5st potential.

Recently, an impressive collection of spectroscopic data has been gathered ${ }^{15-17}$ on the intermolecular vibrations of both $\left(\mathrm{H}_{2} \mathrm{O}\right)_{2}$ and $\left(\mathrm{D}_{2} \mathrm{O}\right)_{2}$. The data set for $\left(\mathrm{D}_{2} \mathrm{O}\right)_{2}$ is more complete than for $\left(\mathrm{H}_{2} \mathrm{O}\right)_{2}$ because, for various reasons given in Ref. 17, the vibrationally excited states of the latter system are harder to access. These vibrations of the whole water molecules in the dimer probe the shape of the wells in the six-dimensional water pair potential in various directions, while the ground state tunneling frequencies which we discussed up to now and used in the tuning of the SAPT- $5 \mathrm{~s}$ potential are sensitive to the heights and widths of the barriers between these wells. The VRT levels corresponding to several of these intermolecular vibrations of $\left(\mathrm{D}_{2} \mathrm{O}\right)_{2}$ were used in the fit of the VRT(ASP-W) potential. ${ }^{37}$ Table V and Fig. 5 contain the ground state and vibrationally excited VRT levels of $\left(\mathrm{H}_{2} \mathrm{O}\right)_{2}$ obtained from the SAPT-5st potential, as well as the experimental data, while Table VI and Fig. 6 contain the $\left(\mathrm{D}_{2} \mathrm{O}\right)_{2}$ data.

Observe that the SAPT-5st potential reproduces accurately not only the ground state tunneling splittings shown in 


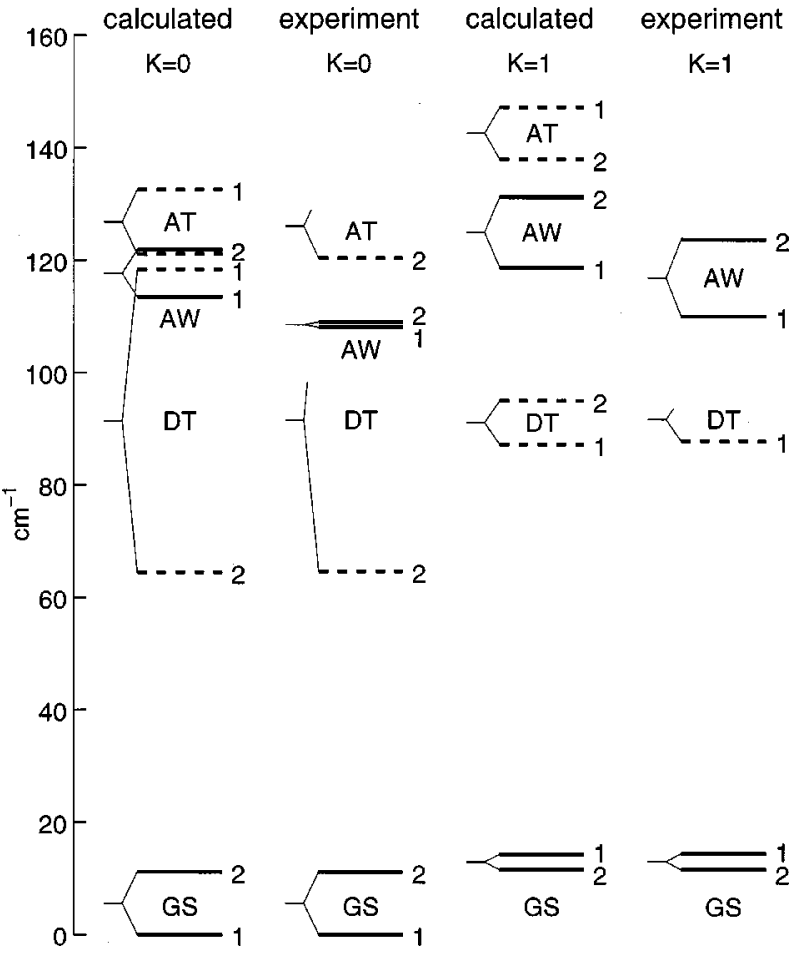

FIG. 5. VRT levels of the $\mathrm{H}_{2} \mathrm{O}$ dimer corresponding to the intermolecular vibrations, calculated from the SAPT-5st potential, in comparison with experimental data (Refs. 15 and 17). The levels 1 and 2 are the origins, $o_{1}$ and $o_{2}$, of the $A_{1}, E_{1}, B_{1}$ and $A_{2}, E_{2}, B_{2}$ levels, respectively. The abbreviations GS, DT, AW, and AT denote the ground state $\left(A^{\prime}\right)$, donor torsion $\left(A^{\prime \prime}\right)$, acceptor wag $\left(A^{\prime}\right)$, and acceptor twist $\left(A^{\prime \prime}\right)$ modes, following Ref. 17. Solid lines refer to $A^{\prime}$ symmetry, dashed lines to $A^{\prime \prime}$ symmetry, with respect to the point group $C_{s}$ of the equilibrium structure. Experimental levels not shown have not been measured to date.

Figs. 3 and 4, but also the frequencies of the intermolecular vibrations. The donor torsion, acceptor twist, and in-plane bending frequencies agree with experiment to within 1 to 3 $\mathrm{cm}^{-1}$, i.e., to about $2 \%$, both for $\left(\mathrm{H}_{2} \mathrm{O}\right)_{2}$ and for $\left(\mathrm{D}_{2} \mathrm{O}\right)_{2}$, for $K=0$ and $K=1$. Also the acceptor tunneling splittings $a(K)$ are in good agreement with experiment for these intermolecular modes. The calculations predict very well, for example, the fact that $a(K)$ in the donor torsion excited state is almost 5 times larger than in the ground state for $\left(\mathrm{H}_{2} \mathrm{O}\right)_{2}$, and about 10 times for $\left(\mathrm{D}_{2} \mathrm{O}\right)_{2}$. The mode that shows the largest deviation from experiment, frequency differences of about $8 \%$ in both $\left(\mathrm{H}_{2} \mathrm{O}\right)_{2}$ and $\left(\mathrm{D}_{2} \mathrm{O}\right)_{2}$, is the acceptor wag. Also $a(K)$ is considerably too large for the $K=0$ component of this excited state, whereas it is more realistic for the $K$ $=1$ component. The in-plane bending mode in $\left(\mathrm{H}_{2} \mathrm{O}\right)_{2}$ is omitted from these considerations, because the assigment of the small number of experimental lines that have been ascribed to this transition is still quite uncertain; ${ }^{17}$ an alternative assignment based on the present calculations is now considered. ${ }^{66}$ The finding that this would be the only case where the experimental end-over-end rotational constant $B$ $+C$ were larger than our calculated value supports our doubt on the assignment of the measured lines to the in-plane bending mode.

In Table VII we compare harmonic vibrational modes, obtained from the SAPT-5s and SAPT-5st potentials, with
TABLE VI. VRT levels, tunneling splittings, and rotational constants (in $\left.\mathrm{cm}^{-1}\right)$ of $\left(\mathrm{D}_{2} \mathrm{O}\right)_{2}$ calculated from SAPT-5st. The numbers in parentheses are experimental values from Refs. 6, 11, 13, and 16.

\begin{tabular}{|c|c|c|c|c|c|c|c|}
\hline & $o_{1}$ & $o_{2}{ }^{\mathrm{a}}$ & $a$ & $i_{1}$ & $i_{2}$ & $B+C$ & $A$ \\
\hline \multicolumn{8}{|c|}{ Ground state $\left(A^{\prime}\right)$} \\
\hline$K=0$ & $\begin{array}{c}0 \\
(0)\end{array}$ & $\begin{array}{c}1.69 \\
(1.77)\end{array}$ & $\begin{array}{c}1.69 \\
(1.77)\end{array}$ & $\begin{array}{c}0.0393 \\
(0.0391)\end{array}$ & $\begin{array}{c}0.0385 \\
(0.0361)\end{array}$ & $\begin{array}{c}0.3759 \\
(0.3622)\end{array}$ & \\
\hline$K=1$ & $\begin{array}{c}5.08 \\
(5.36)\end{array}$ & $\begin{array}{c}4.53 \\
(4.74)\end{array}$ & $\begin{array}{c}0.55 \\
(0.62)\end{array}$ & $\begin{array}{c}0.0383 \\
(0.0359)\end{array}$ & $\begin{array}{c}0.0351 \\
(0.0331)\end{array}$ & $\begin{array}{c}0.3759 \\
(0.3621)\end{array}$ & $\begin{array}{c}4.15 \\
(4.19)\end{array}$ \\
\hline \multicolumn{8}{|c|}{ Donor torsion $\left(A^{\prime \prime}\right)$} \\
\hline$K=0$ & $\begin{array}{c}76.26 \\
(75.38)\end{array}$ & $\begin{array}{c}60.13 \\
(59.59)\end{array}$ & $\begin{array}{c}16.13 \\
(15.81)\end{array}$ & $\begin{array}{c}0.1031 \\
(0.3282)\end{array}$ & $\begin{array}{c}0.1095 \\
(0.2032)\end{array}$ & $\begin{array}{c}0.3742 \\
(0.3622)\end{array}$ & \\
\hline$K=1$ & $\begin{array}{c}68.28 \\
(68.27)\end{array}$ & $\begin{array}{c}72.76 \\
(71.81)\end{array}$ & $\begin{array}{c}4.49 \\
(3.54)\end{array}$ & $\begin{array}{c}0.0517 \\
(0.1318)\end{array}$ & $\begin{array}{c}0.1386 \\
(0.2567)\end{array}$ & $\begin{array}{c}0.3737 \\
(0.3600)\end{array}$ & $\begin{array}{c}2.51 \\
(2.74)\end{array}$ \\
\hline \multicolumn{8}{|c|}{ Acceptor wag $\left(A^{\prime}\right)$} \\
\hline$K=0$ & $\begin{array}{c}89.82 \\
(82.64)\end{array}$ & $\begin{array}{c}95.03 \\
(84.40)\end{array}$ & $\begin{array}{c}5.21 \\
(1.77)\end{array}$ & $\begin{array}{c}0.1508 \\
(0.1310)\end{array}$ & $\begin{array}{c}0.2278 \\
(0.1117)\end{array}$ & $\begin{array}{c}0.3733 \\
(0.3603)\end{array}$ & \\
\hline$K=1$ & $\begin{array}{c}92.74 \\
(85.57)\end{array}$ & $\begin{array}{c}95.31 \\
(89.56)\end{array}$ & $\begin{array}{c}2.57 \\
(4.00)\end{array}$ & $\begin{array}{c}0.7593 \\
(0.3982)\end{array}$ & $\begin{array}{c}0.5474 \\
(0.1676)\end{array}$ & $\begin{array}{c}0.3753 \\
(0.3592)\end{array}$ & $\begin{array}{c}1.79 \\
(4.22)\end{array}$ \\
\hline \multicolumn{8}{|c|}{ Acceptor twist $\left(A^{\prime \prime}\right)$} \\
\hline$K=0$ & $\begin{array}{l}93.78 \\
(92.91)\end{array}$ & $\begin{array}{c}91.98 \\
(90.37)\end{array}$ & $\begin{array}{c}1.80 \\
(2.54)\end{array}$ & $\begin{array}{c}0.8407 \\
(0.4320)\end{array}$ & $\begin{array}{c}0.9965 \\
(0.4432)\end{array}$ & $\begin{array}{c}0.3767 \\
(0.3665)\end{array}$ & \\
\hline$K=1$ & 103.34 & 99.92 & 3.42 & 0.4334 & 0.6055 & 0.3751 & 8.94 \\
\hline \multicolumn{8}{|c|}{ In-plane bend $\left(A^{\prime}\right)$} \\
\hline$K=0$ & $\begin{array}{c}107.32 \\
(104.24)\end{array}$ & 140.50 & 33.19 & $\begin{array}{c}0.6905 \\
(0.7832)\end{array}$ & 0.1312 & $\begin{array}{c}0.3725 \\
(0.3632)\end{array}$ & \\
\hline$K=1$ & 127.47 & 119.43 & 8.04 & 0.3265 & 0.0551 & & -0.27 \\
\hline \multicolumn{8}{|c|}{ Stretch $\left(A^{\prime}\right)$} \\
\hline$K=0$ & 144.44 & 150.88 & 6.44 & 1.399 & 0.0175 & 0.3701 & \\
\hline
\end{tabular}

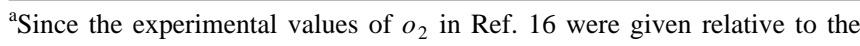
ground state value of $o_{2}$, we added the experimental estimate for the ground state acceptor splitting $a(K=0)=53 \mathrm{GHz}=1.7679 \mathrm{~cm}^{-1}$ to all experimental values.

the exact SAPT-5st frequencies. In labeling the vibrational levels we followed the tentative assignment of Saykally and co-workers. ${ }^{15-17}$ We observe that the harmonic values are too high by a factor ranging from 1.2 to 1.4 , except in the case of the in-plane bend mode. For this mode the factor is 2.6, which casts serious doubt on the assignment of the transition at $139.83 \mathrm{~cm}^{-1}$ to the in-plane bend mode. An analysis of the exact anharmonic wave functions to establish the character of the intermolecular vibrations of the water dimer is in progress. Notice that the harmonic frequencies, especially those from SAPT-5st, agree quite well with the harmonic frequencies obtained from $a b$ initio calculations ${ }^{48}$ with the second order Møller-Plesset (MP2) method.

The small donor-acceptor interchange splittings $i_{1}$ and $i_{2}$ agree not so well with experiment in the vibrationally excited states as they do in the ground state: deviations by a factor of 2 to 3 are typical. Still, the observation that for particular vibrationally excited states $i_{1}$ and $i_{2}$ are 10 to 20 times larger than in the ground state is qualitatively reproduced by our calculations. The calculated end-over-end rota- 


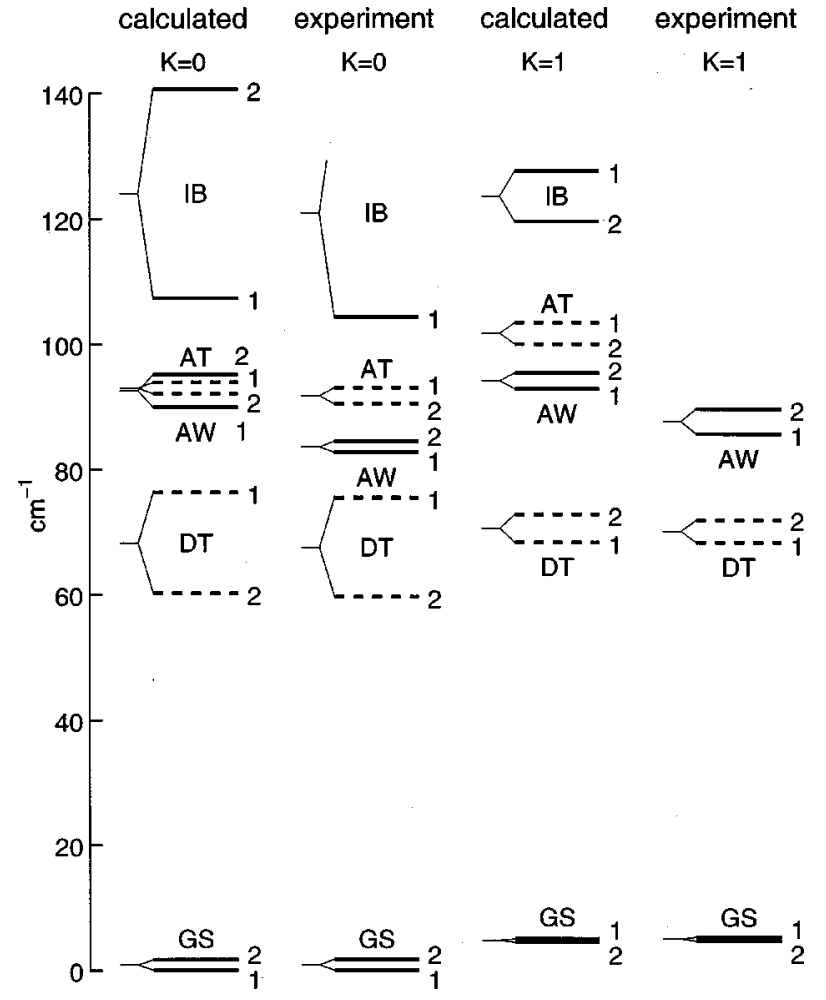

FIG. 6. VRT levels of the $\mathrm{D}_{2} \mathrm{O}$ dimer corresponding to the intermolecular vibrations, calculated from the SAPT-5st potential, in comparison with experimental data (Refs. 15 and 16). All symbols are explained in Fig. 5, except for IB which denotes the in-plane bend $\left(A^{\prime}\right)$ mode.

tional constant $B+C$ is systematically somewhat too large. This is an effect of the tuning: the $B+C$ value from $\mathrm{SAPT}-5 \mathrm{~s}$ was closer to the experimental value. The (much larger) calculated values for the long-axis rotational constant $A$ are generally in good agreement with experiment, with the exception (again) of the acceptor wagging mode in $\left(\mathrm{D}_{2} \mathrm{O}\right)_{2}$. One may find it strange that the $A$ values obtained from $K$ $=1$ and $K=0$ energy level differences $\Delta$, cf. Figs. 3 and 4 , are sometimes very large and sometimes negative. This indicates that $A$ is not simply a component of the inverse rigidrotor inertia tensor, but is affected by the internal motions in the water dimer. A similar phenomenon was observed for the ammonia dimer. ${ }^{55-57}$

TABLE VII. Vibrational frequencies $\left(\mathrm{cm}^{-1}\right)$ for $\left(\mathrm{H}_{2} \mathrm{O}\right)_{2}$. Compared are the numbers in the harmonic approximation from the SAPT-5s and SAPT-5st potential and exact numbers $\left[\left(o_{1}+o_{2}\right) / 2\right.$ for $\left.K=0\right]$ obtained from the SAPT-5st potential. The assignment of the vibrational modes follows Ref. 17. The first column contains harmonic frequencies calculated $a b$ initio (Ref. 48) by the MP2 method.

\begin{tabular}{lcccc}
\hline \hline \multicolumn{1}{c}{ Mode } & $\begin{array}{c}\text { Harmonic } \\
\text { MP2 }\end{array}$ & $\begin{array}{c}\text { Harmonic } \\
\text { SAPT-5s }\end{array}$ & $\begin{array}{c}\text { Harmonic } \\
\text { SAPT-5st }\end{array}$ & $\begin{array}{c}\text { exact } \\
\text { SAPT-5st }\end{array}$ \\
\hline Donor torsion $\left(A^{\prime \prime}\right)$ & 140 & 110.72 & 121.00 & 85.72 \\
Acceptor wag $\left(A^{\prime}\right)$ & 157 & 140.64 & 157.87 & 111.98 \\
Acceptor twist $\left(A^{\prime \prime}\right)$ & 145 & 127.04 & 143.70 & 121.11 \\
Stretch $\left(A^{\prime}\right)$ & 186 & 172.61 & 186.83 & $\ldots$ \\
In-plane bend $\left(A^{\prime}\right)$ & 354 & 342.69 & 369.99 & 139.83 \\
Out-of-plane bend $\left(A^{\prime \prime}\right)$ & 645 & 543.87 & 564.69 & $\ldots$ \\
\hline \hline
\end{tabular}

We illustrate the effect of the tuning on the frequencies of the intermolecular vibrations by quoting the values of the vibrational band origins (in $\mathrm{cm}^{-1}$ for $J=K=0$ ) computed for $\left(\mathrm{D}_{2} \mathrm{O}\right)_{2}$ with the $a b$ initio SAPT-5s potential: $o_{1}$ $=72.90, o_{2}=47.55$ for the donor torsion, $o_{1}=80.28, o_{2}$ $=86.03$ for the acceptor wag, and $o_{1}=89.44, o_{2}=85.27$ for the acceptor twist. When comparing these values to those in Table VI, one observes that the acceptor wag mode is better represented by the $a b$ initio SAPT-5s potential, whereas the donor torsion and acceptor twist frequencies agree markedly better with experiment after the tuning. On the whole, we may conclude that the intermolecular vibrations from SAPT-5s are of good quality, but that the tuning clearly improves agreement with experiment for most of the vibrationally excited levels, as it did for the ground state. This is remarkable, because the tuning procedure involves only one ground state tunneling splitting, just for $\left(\mathrm{H}_{2} \mathrm{O}\right)_{2}$. Summarizing our results for the intermolecular vibrations, we may say that the ground as well as the excited VRT levels of both $\left(\mathrm{H}_{2} \mathrm{O}\right)_{2}$ and $\left(\mathrm{D}_{2} \mathrm{O}\right)_{2}$ are very well represented by the SAPT5 st potential.

Another aspect of the present work is that it provides a more complete characterization of the VRT levels of the water dimer. The experimentally determined transitions are sufficient to fix most, but not all of the levels. We already mentioned that in $\left(\mathrm{H}_{2} \mathrm{O}\right)_{2}$ the sum $a(K=0)+a(K=1)=13.92$ $\mathrm{cm}^{-1}$ has been measured, but that the individual values of the acceptor tunneling splittings for $K=0$ and $K=1$ were not known. The value of $a(K=0)=9.4 \mathrm{~cm}^{-1}$ first given in the experimental paper of Zwart et al., ${ }^{7}$ and later quoted $^{33,14,34}$ as the experimental value, was actually extracted from a fit of the spectroscopic data with the model of Coudert and Hougen. ${ }^{44,45}$ This model is approximate and, moreover, is based on the early and rather poor potential of Ref. 46. A more precise value, $a(K=0)=11.2 \mathrm{~cm}^{-1}$, is obtained from our six-dimensional calculations with the SAPT5 st potential which reproduce the measured quantity $a(K$ $=0)+a(K=1)=13.9 \mathrm{~cm}^{-1}$. While one must conclude that the Coudert-Hougen model is not so reliable for the large acceptor tunneling splittings, the smaller splittings originating from donor-acceptor interchange and the shifts from bifurcation tunneling turn out to be accurately represented by this model. Also for $\left(\mathrm{D}_{2} \mathrm{O}\right)_{2}$ the experimental value of $a(K$ $=0)=1.77 \mathrm{~cm}^{-1}$ was not directly measured; it was based on the assumption ${ }^{13}$ that the value of $a(K=0)$ for the acceptor antisymmetric intramolecular $\mathrm{O}-\mathrm{D}$ stretch excited state of $\left(\mathrm{D}_{2} \mathrm{O}\right)_{2}$ is equal to the ground state value. Our calculations demonstrate that this assumption is justified.

Finally, we discuss some properties of the SAPT-5st potential, in relation to the SAPT-5s potential described in paper I. ${ }^{38}$ The Fortran code that generates both potentials is deposited as AIP Document No. EPAPS: EPRLTAO-84060018. The well depth $D_{e}$ is $4.86 \mathrm{kcal} / \mathrm{mol}$ for SAPT-5s and $5.03 \mathrm{kcal} / \mathrm{mol}$ for SAPT-5st. The most reliable estimate, from the ab initio work of Klopper et al. ${ }^{67}$ is $D_{e}=5.02$ $\pm 0.05 \mathrm{kcal} / \mathrm{mol}$. The latter value is computed for monomers at their (relaxed) equilibrium configurations, see paper I for a more detailed discussion. Obviously, the tuning gives an improvement of the well depth. The dimer dissociation energy 
$D_{0}$ of $\left(\mathrm{H}_{2} \mathrm{O}\right)_{2}$ is $3.05 \mathrm{kcal} / \mathrm{mol}=1067 \mathrm{~cm}^{-1}$ with SAPT- $5 \mathrm{~s}$ and $3.08 \mathrm{kcal} / \mathrm{mol}=1077 \mathrm{~cm}^{-1}$ with SAPT-5st. For $\left(\mathrm{D}_{2} \mathrm{O}\right)_{2}$ SAPT-5st yields $D_{0}=3.47 \mathrm{kcal} / \mathrm{mol}=1214 \mathrm{~cm}^{-1}$. These $D_{0}$ values refer to the ground state of $A_{1}^{+}$symmetry, i.e., to the para-para dimer of $\mathrm{H}_{2} \mathrm{O}$ and the ortho-ortho dimer of $\mathrm{D}_{2} \mathrm{O}$. The best experimental value ${ }^{68}$ of $D_{0}$ for $\left(\mathrm{H}_{2} \mathrm{O}\right)_{2}$ is 1168 $\pm 245 \mathrm{~cm}^{-1}$, see paper I. The second virial coefficients computed with both SAPT-5s potentials do not differ much; ${ }^{38}$ they are in good agreement with the best experimental data.

The water dimer equilibrium geometry [all angles and distances refer to the center of mass (c.m.) origins] calculated with SAPT-5s has $R_{e}=5.60 \mathrm{bohr}$, an angle $\theta_{A}$ $=119.2^{\circ}$ between the donor $C_{2}$ axis and the intermolecular vector $\vec{R}_{A B}$ (corresponding to the H-c.m. - c.m. angle $\alpha$ $\left.=5.2^{\circ}\right)$ and an angle $\theta_{B}=129.3^{\circ}$ between $\vec{R}_{A B}$ and the acceptor $C_{2}$ axis. The SAPT-5st potential has the somewhat smaller $R_{e}$ value of $5.53 \mathrm{bohr}$, nearly the same angle $\theta_{A}$ $=118.5^{\circ}\left(\alpha=5.9^{\circ}\right)$, and a somewhat smaller acceptor angle $\theta_{B}=123.7^{\circ}$. The smaller value of $R_{e}$ explains the larger endover-end rotational constant $B+C$, which actually has become slightly too large in comparison with experiment. Apparently, the tuning of the SAPT-5s potential which increases the well depth $D_{e}$ shifts the $R_{e}$ value to a distance that is somewhat too short. This is probably related to our choice to tune merely the linear parameters in the exponential exchange repulsion.

In paper I and in Sec. II of the present paper we discussed the energy barriers corresponding to the various tunneling processes. In SAPT-5s these barriers are $156 \mathrm{~cm}^{-1}$ for acceptor tunneling, $185 \mathrm{~cm}^{-1}$ for donor-acceptor interchange, and $636 \mathrm{~cm}^{-1}$ for bifurcation tunneling. The barriers in SAPT-5st are 222, 248, and $685 \mathrm{~cm}^{-1}$, respectively. The increase of the acceptor tunneling barrier is accompanied by a substantial reduction of the corresponding tunneling splitting $a(K)$, as might be expected, but the increase of the interchange and bifurcation barrier heights has almost no effect on the tunneling splittings $i_{1}, i_{2}$ and shifts $b_{1}, b_{2}$. In general, it is obvious that tunneling splittings and shifts depend not only on the heights, but also on the widths of the barriers. Moreover, tunneling in the water dimer is not a one-dimensional process: all six intermolecular degrees of freedom are strongly coupled and we must conclude that the energy level splittings are the result of a subtle balance between various effects.

\section{CONCLUSION}

This paper presents a method and its computer implementation to obtain nearly exact quantum levels and wave functions of the water dimer from a six-dimensional intermolecular potential. This method is applied to the $a b$ initio SAPT-5s potential presented in paper I. It is found that this potential passes the very critical test of quantitatively reproducing detailed dimer spectroscopic data better than all previous $a b$ initio and (semi-)empirical water pair potentials, with the exception of the VRT(ASP-W) potential ${ }^{37}$ which was fit to these data. A tuning procedure was developed to use the experimental VRT levels to improve the SAPT-5s potential. This procedure takes into account that one quan- tity, the ground state acceptor tunneling splitting, needed improvement, whereas other quantities derived from the SAPT-5s potential are already in very good agreement with the spectroscopic data. Another aspect of the procedure is that it takes care that the tuned potential deviates as little as possible from the original ab initio potential. The resulting SAPT-5st potential gives excellent agreement with experiment, not only for the ground state VRT levels of $\left(\mathrm{H}_{2} \mathrm{O}\right)_{2}$ that were used in the tuning, but also for the much smaller ground state tunneling splittings in $\left(\mathrm{D}_{2} \mathrm{O}\right)_{2}$ and for the large set of spectroscopic data that has recently become available for the excited VRT levels of both $\left(\mathrm{H}_{2} \mathrm{O}\right)_{2}$ and $\left(\mathrm{D}_{2} \mathrm{O}\right)_{2}$ corresponding to the intermolecular vibrations. Hence, we may conclude that SAPT-5st is an accurate water pair potential.

In calculations on trimers of $\mathrm{H}_{2} \mathrm{O}$ and $\mathrm{D}_{2} \mathrm{O}^{35}$ it was demonstrated that the SAPT-5st potential, when supplemented with accurately calculated three-body interactions, reproduces also the measured torsional (flipping) frequencies in the water trimer. It is clearly superior to the empirical VRT(ASP-W) potential fit to the dimer spectrum in that respect. Thus, SAPT-5st seems to be a more realistic pair potential in regions not probed by the dimer spectrum. The reason for that is probably that the $a b$ initio SAPT-5s potential is of better quality than the ASP-W potential ${ }^{53}$ on which the VRT(ASP-W) potential was based.

\section{ACKNOWLEDGMENTS}

We thank R. J. Saykally for stimulating discussions and for making available their experimental results before publication. E.M.M., R.B., and K.S. would like to acknowledge support by NSF Grants Nos. CHE-9626739, CHE-9982134, and ARO AASERT.

\section{APPENDIX: TUNING ALGORITHMS}

In order to solve Eq. (25) while minimizing $B$ [Eq. (23)] we determine the generalized singular value decomposition $(\text { GSVD })^{69}$ of the matrices $\mathbf{D}$ and $\mathbf{S}^{1 / 2}$. We apply the GSVD algorithm implemented in the Matlab package. ${ }^{70}$ Since $\mathbf{S}$ is positive definite, its square root may be readily obtained from its eigenvalue decomposition. The GSVD factorizes the two matrices $\mathbf{D}$ and $\mathbf{S}^{1 / 2}$ simultaneously:

$$
\begin{aligned}
& \mathbf{D}=\mathbf{U P X}^{T}, \\
& \mathbf{S}^{1 / 2}=\mathbf{V Q X}^{T},
\end{aligned}
$$

where $\mathbf{U}$ and $\mathbf{V}$ are (real) unitary matrices, $\mathbf{P}$ is a rectangular matrix with $P_{i i} \geqslant 0$ and all other elements equal to zero, and $\mathbf{Q}$ a square non-negative diagonal matrix. Uniqueness of solution requires $\mathbf{P}^{T} \mathbf{P}+\mathbf{Q}^{T} \mathbf{Q}=\mathbf{1}$. Since in our problem the matrix $\mathbf{S}$ has full rank, also $\mathbf{X}$ must have full rank and $\mathbf{X}^{-T}$ $\equiv\left(\mathbf{X}^{T}\right)^{-1}$ must exist. Thus, if the matrix $\mathbf{D}$ has $n_{c}$ linearly independent rows, the matrix $\mathbf{P}$ must have precisely $n_{c}$ nonzero diagonal elements since its rank must be equal to the rank $n_{c}$ of the matrix $\mathbf{U}^{T} \mathbf{D} \mathbf{X}^{-T}$. With the definition

$$
\mathbf{y} \equiv \mathbf{X}^{T} \Delta \mathbf{p}
$$

Eq. (20) may be rewritten as

$$
\mathbf{P y}=\mathbf{U}^{T} \mathbf{d} \text {. }
$$


This equation fixes $n_{c}$ components of the vector $\mathbf{y}$. From Eqs. (23), (A1), (A2) and the unitarity of $\mathbf{V}$ we find

$$
B=\Delta \mathbf{p}^{T} \mathbf{S} \Delta \mathbf{p}=\left\|\mathbf{S}^{1 / 2} \Delta \mathbf{p}\right\|^{2}=\|\mathbf{Q} \mathbf{y}\|^{2}=\sum_{i} Q_{i i}^{2} y_{i}^{2} .
$$

Thus, the remaining components of the vector $\mathbf{y}$ should be set to zero in order to minimize $B$. Finally $\Delta \mathbf{p}$ is determined by solving Eq. (A2). The condition number of the matrix $\mathbf{X}$ is on the order of $10^{9}$.

Readers familiar with the (G)SVD method may be surprised that its application is advantageous in this case, even though no singular vectors corresponding to small singular values are eliminated. Analysis of the algorithm used here shows that the crucial step from a numerical point of view is the computation of the square root of the $S$ matrix. This computation is unproblematic even if $\mathbf{S}$ is singular. The fact that our procedure is to be preferred over the direct calculation of $\Delta \mathbf{p}$ from Eq. (25) is demonstrated by our value of 0.29 atomic units for $B(\Delta \mathbf{p})$ in Eq. (23), whereas the computation of $\Delta \mathbf{p}$ by Eq. (25) yields a minimum value of 0.42 . The CPU-time required for these calculations is negligible except for the calculation of the matrices $\mathbf{A}$ [Eq. (16)] and $\mathbf{S}$ [Eq. (24)]. With the method described here, the CPU-time required can be limited to roughly the cost of a single Davidson iteration of $\mathbf{H x}$, see Eq. (13), for each state $\psi_{k}$.

We now turn to the computation of the matrix A defined by Eq. (16). For the expanded potential we have

$$
\frac{\partial V}{\partial p_{i}}=\sum_{\Lambda} \frac{\partial v_{\Lambda}(R)}{\partial p_{i}} A_{\Lambda}\left(\boldsymbol{\omega}_{A}, \boldsymbol{\omega}_{B}\right),
$$

where $v_{\Lambda}(R)$ is given in Eq. (5). Note that for the linear parameters all $\partial V / \partial p_{i}$ may be calculated on the grid with a slightly modified implementation of the SAPT-5s routine that returns the individual terms. Substituting the expansion

$$
\psi_{k}=\sum_{n, \mathbf{I}}\left|\Phi_{n}(R)\right\rangle|\mathbf{I} ; J M\rangle c_{n \mathbf{I} ; k}
$$

and Eq. (5) into Eq. (16) gives

$$
\begin{aligned}
A_{k i}= & \sum_{n^{\prime} \mathbf{I}^{\prime} n \mathbf{I} \mathbf{\Lambda}}\left\langle\Phi_{n^{\prime}}(R)\left|\frac{\partial v_{\boldsymbol{\Lambda}}(R)}{\partial p_{i}}\right| \Phi_{n}(R)\right\rangle \\
& \times\left\langle\mathbf{I}^{\prime} ; J M\left|A_{\boldsymbol{\Lambda}}\left(\boldsymbol{\omega}_{A}, \boldsymbol{\omega}_{B}\right)\right| \mathbf{I} ; J M\right\rangle c_{n^{\prime} \mathbf{I}^{\prime} ; k} c_{n \mathbf{I} ; k} \\
= & \sum_{n^{\prime} n \boldsymbol{\Lambda}} y_{i}\left(n^{\prime}, n, \boldsymbol{\Lambda}\right) z_{k}\left(n^{\prime}, n, \boldsymbol{\Lambda}\right)
\end{aligned}
$$

where

$$
y_{i}\left(n^{\prime}, n, \boldsymbol{\Lambda}\right)=\left\langle\Phi_{n^{\prime}}(R)\left|\frac{\partial v_{\boldsymbol{\Lambda}}(R)}{\partial p_{i}}\right| \Phi_{n}(R)\right\rangle
$$

and

$z_{k}\left(n^{\prime}, n, \boldsymbol{\Lambda}\right)=\sum_{\mathbf{I}^{\prime}, \mathbf{I}}\left\langle\mathbf{I}^{\prime} ; J M\left|A_{\boldsymbol{\Lambda}}\left(\boldsymbol{\omega}_{A}, \boldsymbol{\omega}_{B}\right)\right| \mathbf{I} ; J M\right\rangle_{n^{\prime} \mathbf{I}^{\prime} ; k} c_{n \mathbf{I} ; k}$

This last factor, which is the most expensive one, may be readily calculated by a single call (for each vector $k$ ) to a slightly modified version of the subroutine $\mathbf{H x}$, which leaves out the kinetic energy and stores the contributions for each $n^{\prime}, n$ and $\Lambda$ separately.

${ }^{1}$ T. R. Dyke and J. S. Muenter, J. Chem. Phys. 60, 2929 (1974).

${ }^{2}$ T. R. Dyke, K. M. Mack, and J. S. Muenter, J. Chem. Phys. 66, 498 (1977).

${ }^{3}$ J. A. Odutola et al., J. Chem. Phys. 88, 5352 (1988).

${ }^{4}$ Z. S. Huang and R. E. Miller, J. Chem. Phys. 88, 8008 (1988).

${ }^{5}$ K. L. Busarow et al., J. Chem. Phys. 90, 3937 (1989).

${ }^{6}$ E. Zwart, J. J. ter Meulen, and W. L. Meerts, Chem. Phys. Lett. 173, 115 (1990).

${ }^{7}$ E. Zwart, J. J. ter Meulen, W. L. Meerts, and L. H. Coudert, J. Mol. Spectrosc. 147, 27 (1991).

${ }^{8}$ G. T. Fraser, Int. Rev. Phys. Chem. 10, 189 (1991).

${ }^{9}$ N. Pugliano and R. J. Saykally, J. Chem. Phys. 96, 1832 (1992).

${ }^{10}$ N. Pugliano, J. D. Cruzan, J. G. Loeser, and R. J. Saykally, J. Chem. Phys. 98, 6600 (1993).

${ }^{11}$ E. N. Karyakin, G. T. Fraser, and R. D. Suenram, Mol. Phys. 78, 1179 (1993).

${ }^{12}$ E. N. Karyakin et al., J. Chem. Phys. 102, 1114 (1995).

${ }^{13}$ J. B. Paul, R. A. Provencal, and R. J. Saykally, J. Phys. Chem. A 102, 3279 (1998).

${ }^{14}$ R. S. Fellers, L. B. Braly, R. J. Saykally, and C. Leforestier, J. Chem. Phys. 110, 6306 (1999).

${ }^{15}$ L. B. Braly, Ph.D. thesis, University of California at Berkeley, 1999.

${ }^{16}$ L. B. Braly et al., J. Chem. Phys. 112, 10293 (2000).

${ }^{17}$ L. B. Braly et al., J. Chem. Phys. 112, 10314 (2000).

${ }^{18}$ K. Liu et al., J. Am. Chem. Soc. 116, 3507 (1994).

${ }^{19}$ M. R. Viant et al., J. Phys. Chem. A 101, 9032 (1997).

${ }^{20}$ M. R. Viant et al., J. Chem. Phys. 110, 4369 (1999).

${ }^{21}$ M. G. Brown et al., J. Chem. Phys. 111, 7789 (1999).

${ }^{22}$ J. D. Cruzan, M. R. Viant, M. G. Brown, and R. J. Saykally, J. Phys. Chem. A 101, 9022 (1997).

${ }^{23}$ K. Liu, M. G. Brown, J. D. Cruzan, and R. J. Saykally, J. Phys. Chem. A 101, 9011 (1997).

${ }^{24}$ M. G. Brown, F. N. Keutsch, and R. J. Saykally, J. Chem. Phys. 109, 9645 (1998).

${ }^{25}$ K. Liu, M. G. Brown, and R. J. Saykally, J. Phys. Chem. A 101, 8995 (1997).

${ }^{26}$ F. Huisken, M. Kaloudis, and A. Kulcke, J. Phys. Chem. 104, 17 (1996).

${ }^{27}$ U. Buck et al., Phys. Rev. Lett. 80, 2578 (1998).

${ }^{28}$ J. B. Paul et al., J. Chem. Phys. 109, 10201 (1998).

${ }^{29}$ J. Brudermann et al., J. Chem. Phys. 110, 10649 (1999).

${ }^{30}$ A. Luzar and D. Chandler, Phys. Rev. Lett. 76, 928 (1996).

${ }^{31}$ A. Luzar and D. Chandler, Nature (London) 379, 55 (1996).

${ }^{32}$ S. Woutersen, U. Emmerichs, and H. J. Bakker, Science 278, 658 (1997).

${ }^{33}$ C. Leforestier et al., J. Chem. Phys. 106, 8527 (1997).

${ }^{34}$ H. Chen, S. Liu, and J. C. Light, J. Chem. Phys. 110, 168 (1999).

${ }^{35}$ G. C. Groenenboom et al., Phys. Rev. Lett. 84, 4072 (2000).

${ }^{36}$ D. J. Wales, "Rearrangements and tunneling in water clusters," in $\mathrm{Ad}$ vances in Molecular Vibrations and Collision Dynamics, edited by J. M. Bowman and Z. Bačić (JAI, Stamford, 1998).

${ }^{37}$ R. S. Fellers et al., Science 284, 945 (1999).

${ }^{38}$ E. M. Mas et al., J. Chem. Phys. 113, 6687 (2000), preceding paper.

${ }^{39}$ K. Morokuma, J. Chem. Phys. 48, 3275 (1968).

${ }^{40}$ P. A Kollman and L. C. Allen, J. Chem. Phys. 51, 3286 (1969).

${ }^{41}$ J. D. Bene and J. A. Pople, J. Chem. Phys. 52, 1301 (1970).

${ }^{42}$ D. Hankins, J. W. Moskowitz, and F. H. Stillinger, J. Chem. Phys. 53, 4544 (1970).

${ }^{43}$ T. R. Dyke, J. Chem. Phys. 66, 492 (1977).

${ }^{44}$ L. H. Coudert and J. T. Hougen, J. Mol. Spectrosc. 130, 86 (1988).

${ }^{45}$ L. H. Coudert and J. T. Hougen, J. Mol. Spectrosc. 139, 259 (1990).

${ }^{46}$ D. F. Coker and R. O. Watts, J. Phys. Chem. 91, 2513 (1987).

${ }^{47}$ J. T. Hougen, J. Mol. Spectrosc. 114, 395 (1985).

${ }^{48}$ S. S. Xantheas and T. H. Dunning, J. Chem. Phys. 99, 8774 (1993).

${ }^{49}$ S. C. Althorpe and D. C. Clary, J. Chem. Phys. 101, 3603 (1994).

${ }^{50}$ S. C. Althorpe and D. C. Clary, J. Chem. Phys. 102, 4390 (1995).

${ }^{51}$ J. K. Gregory and D. C. Clary, J. Chem. Phys. 102, 7817 (1995).

${ }^{52}$ C. Leforestier, J. Chem. Phys. 101, 7357 (1994).

${ }^{53}$ C. Millot and A. J. Stone, Mol. Phys. 77, 439 (1992).

${ }^{54}$ G. Brocks, A. van der Avoird, B. T. Sutcliffe, and J. Tennyson, Mol. Phys. 50, 1025 (1983). 
${ }^{55}$ A. van der Avoird, P. E. S. Wormer, and R. Moszynski, Chem. Rev. 94, 1931 (1994).

${ }^{56}$ J. W. I. van Bladel, A. van der Avoird, P. E. S. Wormer, and R. J. Saykally, J. Chem. Phys. 97, 4750 (1992).

${ }^{57}$ E. H. T. Olthof, A. van der Avoird, and P. E. S. Wormer, J. Chem. Phys. 101, 8430 (1994).

${ }^{58}$ D. M. Brink and G. R. Satchler, Angular Momentum, 3rd ed. (Clarendon, Oxford, 1993).

${ }^{59}$ D. T. Colbert and W. H. Miller, J. Chem. Phys. 96, 1982 (1992).

${ }^{60}$ G. C. Groenenboom and D. T. Colbert, J. Chem. Phys. 99, 9681 (1993).

${ }^{61}$ E. R. Davidson, J. Comput. Phys. 17, 87 (1975).

${ }^{62}$ G. Herzberg, Molecular Spectra and Molecular Structure, Vol. 2: Infrared and Raman Spectra of Polyatomic Molecules (Van Nostrand, New York, 1945).
${ }^{63}$ S. T. Epstein, The Variation Method in Quantum Chemistry (Academic, New York, 1974).

${ }^{64} \mathrm{C}$. Leforestier (private communication).

${ }^{65}$ E. M. Mas, K. Szalewicz, R. Bukowski, and B. Jeziorski, J. Chem. Phys. 107, 4207 (1997).

${ }^{66} \mathrm{R}$. J. Saykally (private communication).

${ }^{67}$ W. Klopper, J. van Duijneveldt-van de Rijdt, and F. van Duijneveldt, Phys. Chem. Phys. (in press).

${ }^{68}$ L. A. Curtiss, D. J. Frurip, and M. Blander, J. Chem. Phys. 71, 2703 (1979).

${ }^{69}$ G. H. Golub and C. Van Loan, Matrix Computations, 3rd ed. (Johns Hopkins University, Baltimore, 1996).

${ }^{70}$ The MathWorks, Inc., 24 Prime Park Way, Natick, MA, MATLAB Version 5, 1996, http://www.mathworks.com/.

${ }^{71}$ C. J. Keoshian and R. J. Saykally (private communication). 